\title{
Copper(I) Alkoxide-Catalyzed Alkynylation of Trifluoromethyl Ketones
}

Rie Motoki, Motomu Kanai, ${ }^{*}$ and Masakatsu Shibasaki*

General: NMR spectra were recorded on a JEOL JNM-LA500 spectrometer, operating at $500 \mathrm{MHz}$ for ${ }^{1} \mathrm{H} \mathrm{NMR}, 125.65 \mathrm{MHz}$ for ${ }^{13} \mathrm{C}$ NMR, and $470.4 \mathrm{MHz}$ for ${ }^{19} \mathrm{~F}$ NMR. For ${ }^{1} \mathrm{H}$ and ${ }^{13} \mathrm{C}$ NMR, chemical shifts were reported in the scale relative to the solvent used as an internal reference. ${ }^{19} \mathrm{~F}$ NMR was measured using $\mathrm{CF}_{3} \mathrm{CO}_{2} \mathrm{H}$ as an external standard (-79 ppm). Optical rotations were measured on a JASCO P-1010 polarimeter. Column chromatographies were performed with silica gel Merck 60 (230-400 mesh ASTM). The enantiomeric excess (ee) was determined by GC analysis (SHIMADZU GC-14A). In general, reactions were carried out in dry solvents under an argon atmosphere, unless noted otherwise. Normal glassware was used for reactions described in this paper. Aromatic trifluoromethyl ketones $(3 \mathrm{a}, 3 \mathrm{c}-3 \mathrm{~g})$ are commercially available, and used after distillation. Aliphatic trifluoromethyl ketones (3b, 3h) were synthesized following the reported procedure. ${ }^{1}$ (R)-DTBM-SEGPHOS (9) was provided from Takasago International Corporation. Pybox (10) was synthesized following the reported procedure. ${ }^{2}$

A typical procedure for catalytic alkynylation using CuF-phosphine complex (Table 1, entry 1): To a $\mathrm{CuF} \cdot 1 / 2$ benzene $(5 \mathrm{mg}, 0.02 \mathrm{mmol}), \mathrm{KO}{ }^{t} \mathrm{Bu}(2.2 \mathrm{mg}, 0.02 \mathrm{mmol})$ and xantphos (11.6 mg, $0.02 \mathrm{mmol})$ in THF $(0.2 \mathrm{~mL}$ ), trifluoroacetophenone (3a: $28 \mu \mathrm{L}, 0.2$ $\mathrm{mmol}$ ) and alkyne (4a: $44 \mu \mathrm{L}, 0.4 \mathrm{mmol}$ ) were added at room temperature. The mixture was warmed up to $60{ }^{\circ} \mathrm{C}$. After $22 \mathrm{~h}$, the reaction mixture was diluted with AcOEt, and $\mathrm{SiO}_{2}$ was added. Filtration, removal of the solvent gave crude product as brown oil. NMR yield was determined using $\left(\mathrm{CHCl}_{2}\right)_{2}$ as an internal standard. Further purification using $\mathrm{SiO}_{2}(\mathrm{AcOEt} /$ hexane $=1 / 20)$ column chromatography afforded 5aa as pale yellow oil in quantative yield.

A Typical Procedure for Copper Catalyzed Alkynylation of Trifluoromethyl Ketones Using $\mathrm{Cu}$-Diamine Catalyst (Table 3, entry 1): To a toluene solution (0.2 mL) of $\mathrm{Cu}(\mathrm{OTf})_{2}(7.2 \mathrm{mg}, 0.02 \mathrm{mmol}), \mathrm{KO}{ }^{t} \mathrm{Bu}(4.5 \mathrm{mg}, 0.04 \mathrm{mmol})$ and 1,10-phenanthroline (3.6 $\mathrm{mg}, 0.02 \mathrm{mmol}$ ), trifluoromethyl ketone (3a: $28 \mu \mathrm{L}, 0.2 \mathrm{mmol}$ ) and phenylacetylene (4a: $44 \mu \mathrm{L}, 0.4 \mathrm{mmol}$ ) were added at room temperature. Then, the mixture was heated at $100{ }^{\circ} \mathrm{C}$. After $14 \mathrm{hr}$, the reaction mixture was diluted with $\mathrm{AcOEt}$, and $\mathrm{SiO}_{2}$ was added. After filtration, removal of the solvent gave crude product as brown oil. Workup and purification as described above gave the product 5 aa in $95 \%$ yield. The absolute configuration was determined by comparison of the optical rotations to the reported value. 
Spectroscopic data and GC condition of the products:

1,1,1-Trifluoro-4-phenyl-2-phenylbut-3-yn-2-ol (5aa): Racemic compound is known. ${ }^{3}$ $[\alpha]^{26}{ }_{\mathrm{D}}+12.2\left(c 2.25, \mathrm{CHCl}_{3}\right)(42 \%$ ee $)$. CP CHIRALSIL DEX CB, column temperature = $180{ }^{\circ} \mathrm{C}$, injector temperature $=200{ }^{\circ} \mathrm{C}$, detector temperature $=250{ }^{\circ} \mathrm{C}, \mathrm{t}_{\mathrm{R}}=13.7 \mathrm{~min}$ (minor for $S$ isomer) and $14.9 \mathrm{~min}$ (major for $S$ isomer).

1,1,1-Trifluoro-4-phenyl-2- $p$-tolylbut-3-yn-2-ol (5ca): ${ }^{1} \mathrm{H}$ NMR (500 $\left.\mathrm{MHz}, \mathrm{CDCl}_{3}\right) \delta 2.29$

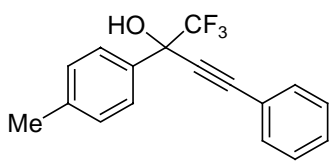
(s, 3H), $3.02(\mathrm{~s}, 1 \mathrm{H}), 7.15(\mathrm{~d}, J=8.0 \mathrm{~Hz}, 2 \mathrm{H}), 7.25^{-7.32}(\mathrm{~m}, 3 \mathrm{H})$, $7.44(\mathrm{~d}, J=6.9 \mathrm{~Hz}, 2 \mathrm{H}), 7.61(\mathrm{~d}, J=8.0 \mathrm{~Hz}, 2 \mathrm{H}) ;{ }^{19} \mathrm{~F} \mathrm{NMR}(466 \mathrm{MHz}$, $\left.\mathrm{CDCl}_{3}\right) \delta-80.2 ;{ }^{13} \mathrm{C} \mathrm{NMR}\left(125 \mathrm{MHz}, \mathrm{CDCl}_{3}\right) \delta 21.1,73.3(\mathrm{q}, J=32.2$ $\mathrm{Hz}$ ), 84.6, 87.9, 121.0, 123.4 (q, $J=284.9 \mathrm{~Hz}$ ), 127.1, 128.4, 129.0, 129.5, 132.0, 132.4; IR (neat, $\mathrm{cm}^{-1}$ ) 3548, 3460, 3034, 2925, 2233, 1184, 821, 757, 734; EI-HRMS Calcd for $\mathrm{C}_{17} \mathrm{H}_{13} \mathrm{~F}_{3} \mathrm{O}^{+}\left(\mathrm{M}^{+}\right)$290.0913, Found 290.0916.

1,1,1-Trifluoro-2-(4-methoxyphenyl)-4-phenylbut-3-yn-2-ol (5da): ${ }^{1} \mathrm{H}$ NMR (500 MHz, $\mathrm{HO} \quad \mathrm{CF}_{3}$ $\left.\mathrm{CDCl}_{3}\right) \delta 3.22(\mathrm{~s}, 1 \mathrm{H}), 3.84(\mathrm{~s}, 3 \mathrm{H}), 6.96(\mathrm{~d}, J=8.6 \mathrm{~Hz}, 2 \mathrm{H})$, $\mathrm{MeO}$ 7.35-7.43 (m, 3H), $7.54(\mathrm{~d}, J=6.9 \mathrm{~Hz}, 2 \mathrm{H}), 7.75(\mathrm{~d}, J=8.6 \mathrm{~Hz}, 2 \mathrm{H})$; $\left.{ }^{19} \mathrm{~F} \mathrm{NMR} \mathrm{(466} \mathrm{MHz,} \mathrm{CDCl}_{3}\right) \delta-80.4 ;{ }^{13} \mathrm{C} \mathrm{NMR}\left(125 \mathrm{MHz} \mathrm{CDCl}_{3}\right) \delta$ $55.3,73.1$ (q, $J=32.6 \mathrm{~Hz}$ ), 84.6, 87.9, 113.5, 121.0, $123.4($ q, $J=285.3 \mathrm{~Hz}$ ), 127.4, 128.4, 128.6, 129.5, 132.0, 160.4; IR (neat, $\mathrm{cm}^{-1}$ ) 3420, 3059, 2938, 2841, 2233, 1170, 758, 757, 690; EI-HRMS Calcd for $\mathrm{C}_{17} \mathrm{H}_{13} \mathrm{~F}_{3} \mathrm{O}_{2}{ }^{+}\left(\mathrm{M}^{+}\right)$306.0862, Found 306.0860.

2-(4-Chlorophenyl)-1,1,1-trifluoro-4-phenylbut-3-yn-2-ol (5ea): ${ }^{1} \mathrm{H}$ NMR (500 MHz, $\mathrm{HO} \quad \mathrm{CF}_{3}$

$\mathrm{Cl}$

$\left.\mathrm{CDCl}_{3}\right) \delta 3.22(\mathrm{~s}, 1 \mathrm{H}), 7.35-7.43(\mathrm{~m}, 3 \mathrm{H}), 7.42(\mathrm{~d}, J=8.6 \mathrm{~Hz}, 2 \mathrm{H})$, $7.53(\mathrm{~d}, J=7.0 \mathrm{~Hz}, 2 \mathrm{H}), 7.75(\mathrm{~d}, J=8.6 \mathrm{~Hz}, 2 \mathrm{H}) ; 19 \mathrm{~F} \mathrm{NMR}(466 \mathrm{MHz}$, $\left.\mathrm{CDCl}_{3}\right) \delta-80.3 ;{ }^{13} \mathrm{C} \mathrm{NMR}\left(125 \mathrm{MHz}, \mathrm{CDCl}_{3}\right) \delta 72.9(\mathrm{q}, J=32.6 \mathrm{~Hz})$, 83.9, 88.4, 120.7, 123.2 (q, $J=285.7 \mathrm{~Hz}$ ), 128.4, 128.5, 128.7, 129.7, 132.0, 133.8, 135.7; IR (neat, $\mathrm{cm}^{-1}$ ) 3547, 3447, 3063, 2234, 1190, 757, 689; EI-HRMS Calcd for $\mathrm{C}_{16} \mathrm{H}_{10} \mathrm{ClF}_{3} \mathrm{O}^{+}\left(\mathrm{M}^{+}\right)$310.0367, Found 310.0367.

2-(4-Bromophenyl)-1,1,1-trifluoro-4-phenylbut-3-yn-2-ol (5fa): ${ }_{1} \mathrm{H}$ NMR (500 MHz, $\mathrm{HO} \quad \mathrm{CF}_{3}$

$\mathrm{Br}$

$\left.\mathrm{CDCl}_{3}\right) \delta 3.21(\mathrm{~s}, 1 \mathrm{H}), 7.35-7.44(\mathrm{~m}, 3 \mathrm{H}), 7.54(\mathrm{~d}, J=6.9 \mathrm{~Hz}, 2 \mathrm{H})$, $7.58(\mathrm{~d}, J=8.6 \mathrm{~Hz}, 2 \mathrm{H}), 7.70(\mathrm{~d}, J=8.6 \mathrm{~Hz}, 2 \mathrm{H}) ;{ }^{19} \mathrm{~F} \mathrm{NMR}(466 \mathrm{MHz}$, $\left.\mathrm{CDCl}_{3}\right) \delta-80.2 ;{ }^{13} \mathrm{C} \mathrm{NMR}\left(125 \mathrm{MHz}, \mathrm{CDCl}_{3}\right) \delta 73.0(\mathrm{q}, J=32.6 \mathrm{~Hz}$ ), 83.9, 88.4, 120.7, 123.2 (q, $J=286.1 \mathrm{~Hz}$ ), 120.7, 124.0, 128.5, 129.0, 129.7, 131.4, 132.1, 134.5; IR (neat, $\mathrm{cm}^{-1}$ ) 3432, 3083, 3060, 2926, 2234, 1188, 824, 757; EI-HRMS Calcd for $\mathrm{C}_{16} \mathrm{H}_{10} \mathrm{BrF}_{3} \mathrm{O}^{+}\left(\mathrm{M}^{+}\right)$353.9862, Found 353.9860.

1,1,1-Trifluoro-4-phenyl-2- $o$-tolylbut-3-yn-2-ol (5ia): $\left.{ }^{1} \mathrm{H} \mathrm{NMR} \mathrm{(500} \mathrm{MHz,} \mathrm{CDCl}_{3}\right) \delta 2.73$ (s, Hо $\left.\mathrm{CF}_{3} \quad 3 \mathrm{H}\right), 3.21(\mathrm{~s}, 1 \mathrm{H}), 7.26-7.45(\mathrm{~m}, 6 \mathrm{H}), 7.54(\mathrm{~d}, J=7.4 \mathrm{~Hz}, 2 \mathrm{H}), 7.89(\mathrm{~d}, J$ $=8.0 \mathrm{~Hz}, 2 \mathrm{H}) ;{ }^{19} \mathrm{~F} \mathrm{NMR}\left(466 \mathrm{MHz}^{\mathrm{CDCl}}{ }_{3}\right) \delta-78.9 ;{ }^{13} \mathrm{C} \mathrm{NMR}(125 \mathrm{MHz}$, 
$\left.\mathrm{CDCl}_{3}\right) \delta 21.7,73.9(\mathrm{q}, J=33.4 \mathrm{~Hz}), 85.0,88.6,121.1,124.0(\mathrm{q}, J=286.1 \mathrm{~Hz}), 125.7$, 128.5, 128.6, 129.3, 129.5, 131.9, 132.5, 132.7, 137.8; IR (neat, $\mathrm{cm}^{-1}$ ) 3541, 3063, 2935, 2231, 1184, 757, 733, 689; EI-HRMS Calcd for $\mathrm{C}_{17} \mathrm{H}_{13} \mathrm{~F}_{3} \mathrm{O}^{+}\left(\mathrm{M}^{+}\right)$290.0913, Found 290.0913.

1,1,1-Trifluoro-4-phenyl-2- $m$-tolylbut-3-yn-2-ol (5ja): ${ }^{1} \mathrm{H} \mathrm{NMR}\left(500 \mathrm{MHz}, \mathrm{CDCl}_{3}\right) \delta 2.43$ Me $\mathrm{HO}_{3} \quad(\mathrm{~s}, 3 \mathrm{H}), 3.22(\mathrm{~s}, 1 \mathrm{H}), 7.26(\mathrm{~d}, J=6.9 \mathrm{~Hz}, 2 \mathrm{H}), 7.32-7.44(\mathrm{~m}, 4 \mathrm{H})$, $7.55(\mathrm{~d}, J=8.0 \mathrm{~Hz}, 2 \mathrm{H}), 7.63(\mathrm{~d}, J=6.3 \mathrm{~Hz}, 2 \mathrm{H}) ; 19 \mathrm{~F} \mathrm{NMR}(466$ $\left.\mathrm{MHz} \mathrm{CDCl}_{3}\right) \delta-80.1 ;{ }^{13} \mathrm{C} \mathrm{NMR}\left(125 \mathrm{MHz} \mathrm{CDCl}_{3}\right) \delta 21.5,73.3(\mathrm{q}, J$ $=33.4 \mathrm{~Hz}$ ), 84.5, 87.9, 121.0, 123.4 (q, $J=284.9 \mathrm{~Hz}), 124.3,127.6,128.1,128.4,129.5$, 130.3, 132.0, 135.2, 138.0; IR (neat, $\mathrm{cm}^{-1}$ ) 3465, 3059, 2925, 2234, 1188, 835, 757, 730, 690; EI-HRMS Calcd for $\mathrm{C}_{17} \mathrm{H}_{13} \mathrm{~F}_{3} \mathrm{O}^{+}\left(\mathrm{M}^{+}\right)$290.0913, Found 290.0914 .

1,1,1-Trifluoro-4-phenyl-2-(thiophen-3-yl)but-3-yn-2-ol (5ga): ${ }^{1} \mathrm{H} \quad \mathrm{NMR} \quad(500 \mathrm{MHz}$, $\left.\mathrm{HO} \mathrm{CF}_{3} \quad \mathrm{CDCl}_{3}\right) \delta 3.17(\mathrm{~s}, 1 \mathrm{H}), 7.35-7.42(\mathrm{~m}, 5 \mathrm{H}), 7.53(\mathrm{~d}, J=8.0 \mathrm{~Hz}, 2 \mathrm{H}), 7.70(\mathrm{~s}$, $\mathrm{S}$ $1 \mathrm{H}) ;{ }^{19} \mathrm{~F} \mathrm{NMR}\left(466 \mathrm{MHz}^{\mathrm{CDCl}}{ }_{3}\right) \delta-80.4 ;{ }^{13} \mathrm{C} \mathrm{NMR}\left(125 \mathrm{MHz} \mathrm{CDCl}_{3}\right) \delta$ $71.3(\mathrm{q}, J=33.4 \mathrm{~Hz}), 84.0,87.6,120.8,123.2(\mathrm{q}, J=286.1 \mathrm{~Hz}), 125.4$, 126.2 126.4, 128.5, 129.6, 132.0, 136.6; IR (neat, $\mathrm{cm}^{-1}$ ) 3433, 3114, 2926, 2235, 1186, 757, 725; EI-HRMS Calcd for $\mathrm{C}_{14} \mathrm{H}_{9} \mathrm{~F}_{3} \mathrm{OS}^{+}\left(\mathrm{M}^{+}\right)$282.0321, Found 282.0315.

3-Trifluoromethyl-1,5-diphenylpent-1-yn-3-ol (5ba): ${ }^{1} \mathrm{H}$ NMR (500 $\left.\mathrm{MHz}, \mathrm{CDCl}_{3}\right) \delta$ $2.16-2.27(\mathrm{~m}, 2 \mathrm{H}), 2.74(\mathrm{~s}, 1 \mathrm{H}), 3.02(\mathrm{t}, J=8.6 \mathrm{~Hz}, 2 \mathrm{H}), 7.19-7.40$
$(\mathrm{~m}, 8 \mathrm{H}), 7.48(\mathrm{~d}, J=6.9 \mathrm{~Hz}, 2 \mathrm{H}) ; 19 \mathrm{~F} \mathrm{NMR}\left(466 \mathrm{MHz}, \mathrm{CDCl}_{3}\right) \delta$
$-81.3 ;{ }^{13} \mathrm{C} \mathrm{NMR}\left(125 \mathrm{MHz}^{\mathrm{HO}} \mathrm{CDCl}_{3}\right) \delta 29.8,36.6,72.2(\mathrm{q}, J=31.8$ $\mathrm{Hz}$ ), 83.3, 87.9, 121.0, 124.1 (q, $J=284.9 \mathrm{~Hz}$ ), 126.2, 128.46, 128.48, 128.6, 129.4, 132.0, 140.9; IR (neat, cm-1) 3432, 3029, 2939, 2238, 1183, 756, 690; EI-HRMS Calcd for $\mathrm{C}_{18} \mathrm{H}_{15} \mathrm{~F}_{3} \mathrm{O}^{+}\left(\mathrm{M}^{+}\right)$304.1070, Found 304.1073.

3-Trifluoromethyl-1-phenyltridecan-1-yn-3-ol (5ha): ${ }_{1}^{1} \mathrm{H}$ NMR (500 $\left.\mathrm{MHz}, \mathrm{CDCl}_{3}\right) \delta$ Ho $\mathrm{CF}_{3} \quad 0.89-0.91(\mathrm{~m}, 3 \mathrm{H}), 1.25-1.44(\mathrm{~m}, 14 \mathrm{H}), 1.67-1.72(\mathrm{~m}, 2 \mathrm{H})$, 1.87-1.92 (m, 2H), 2.64 (s, $1 \mathrm{H}), 7.31-7.39(\mathrm{~m}, 3 \mathrm{H})$, 7.45-7.50 (m, 2H); $\left.19 \mathrm{~F} \mathrm{NMR} \mathrm{(466} \mathrm{MHz}, \mathrm{CDCl}_{3}\right) \delta-81.4$; ${ }^{13} \mathrm{C}$ NMR (125 MHz, $\left.\mathrm{CDCl}_{3}\right) \delta 14.1,22,7,23.3,29.3,29.44,29.48,29.55,29.57,72.4$ (q, $J$ $=31.0 \mathrm{~Hz}$ ), 83.7, 87.3, 121.2, $124.2(\mathrm{q}, J=286.1 \mathrm{~Hz}), 128.4,129.3,132.0$; IR (neat, $\mathrm{cm}^{-1}$ ) 3407, 2926, 2855, 2237, 2237, 1182, 756, 689; EI-HRMS Calcd for $\mathrm{C}_{20} \mathrm{H}_{27} \mathrm{~F}_{3} \mathrm{O}^{+}\left(\mathrm{M}^{+}\right)$ 340.2009 , Found 340.2005.

1,1,1-Trifluoro-2.6-diphenylhex-3-yn-2-ol (5ab): ${ }^{1} \mathrm{H} \mathrm{NMR}\left(500 \mathrm{MHz}, \mathrm{CDCl}_{3}\right) \delta 2.53$ (t, $J=$ $\underbrace{\mathrm{HO} \quad \mathrm{CF}_{3}}_{\mathrm{Ph}}$ $7.3 \mathrm{~Hz}, 2 \mathrm{H}), 2.79(\mathrm{t}, J=7.3 \mathrm{~Hz}, 2 \mathrm{H}), 2.81(\mathrm{~s}, 1 \mathrm{H}), 7.12-7.17(\mathrm{~m}, 3 \mathrm{H})$, 7.18-7.29 (m, 5H), $7.53(\mathrm{~d}, J=7.6 \mathrm{~Hz}, 2 \mathrm{H}) ; 19 \mathrm{~F} \mathrm{NMR}(466 \mathrm{MHz}$, $\left.\mathrm{CDCl}_{3}\right) \delta-80.4 ;{ }^{13} \mathrm{C} \mathrm{NMR}\left(125 \mathrm{MHz}, \mathrm{CDCl}_{3}\right) \delta 20.8,34.3,72.8$ (q, $J=$ 
$32.2 \mathrm{~Hz}$ ), 77.2, 88.7, $123.4(\mathrm{q}, J=285.5 \mathrm{~Hz}), 126.5,127.2,128.1,128.5,128.6,129.3$, 135.5, 139.9; IR (neat, $\mathrm{cm}^{-1}$ ) 3530, 3030, 2931, 2248, 1173, 761, 698; EI-HRMS Calcd for $\mathrm{C}_{18} \mathrm{H}_{15} \mathrm{~F}_{3} \mathrm{O}^{+}\left(\mathrm{M}^{+}\right)$304.1070, Found 304.1067.

1-Triethylsilyl-3-phenyl-3-trifluoromethylprop-1-yn-3-ol (5ac): ${ }^{1} \mathrm{H}$ NMR (500 MHz, $\left.\mathrm{HO} \mathrm{CF}_{3} \quad \mathrm{CDCl}_{3}\right) \delta 0.60(\mathrm{q}, J=8.0 \mathrm{~Hz}, 6 \mathrm{H}), 0.96(\mathrm{t}, J=8.0 \mathrm{~Hz}, 9 \mathrm{H}), 2.95(\mathrm{~s}, 1 \mathrm{H})$, $\mathrm{SiEt}_{3}$ 7.32-7.36 (m, 3H), 7.66-7.69 (m, 2H); $\left.{ }^{19} \mathrm{~F} \mathrm{NMR} \mathrm{(466} \mathrm{MHz,} \mathrm{CDCl}_{3}\right) \delta$ -80.4; ${ }^{13} \mathrm{C} \mathrm{NMR}\left(125 \mathrm{MHz}, \mathrm{CDCl}_{3}\right) \delta 3.8,7.3,73.0(\mathrm{q}, J=32.3 \mathrm{~Hz}), 92.0,101.4,123.2(\mathrm{q}, J$ $=287.3 \mathrm{~Hz}$ ), 127.2, 128.1, 129.4, 135.1; IR (neat, $\mathrm{cm}^{-1}$ ) 3467, 3074, 2958, 2878, 2178, 1261, 1186, 769, 698; EI-HRMS Calcd for $\mathrm{C}_{16} \mathrm{H}_{21} \mathrm{~F}_{3} \mathrm{OSi}^{+}\left(\mathrm{M}^{+}\right)$314.1308, Found 314.1311 .

4-Triethylsilyl-2-triethylsilyloxy-1,1,1-trifluoromethyl-2-phenylbut-3-yne (Et3Si-5ac): $\mathrm{Et}_{3} \mathrm{SiO} \quad \mathrm{CF}_{3} \quad{ }^{1} \mathrm{H} \mathrm{NMR}\left(500 \mathrm{MHz}, \mathrm{CDCl}_{3}\right) \delta 0.67-0.81(\mathrm{~m}, 12 \mathrm{H}), 0.95(\mathrm{t}, J=7.9 \mathrm{~Hz}$, SiEt $\left._{3} 1 \mathrm{H}\right), 0.95(\mathrm{~d}, J=7.9 \mathrm{~Hz}, 2 \mathrm{H}), 7.38-7.41(\mathrm{~m}, 8 \mathrm{H}), 7.75-7.79(\mathrm{~m}, 2 \mathrm{H}) ; 19 \mathrm{~F}$ NMR (466 MHz, $\left.\mathrm{CDCl}_{3}\right) \delta-80.7 ;{ }^{13} \mathrm{C} \mathrm{NMR}\left(125 \mathrm{MHz} \mathrm{CDCl}_{3}\right) \delta 4.0,5.8$, 6.6, 7.3, 74.3 (q, $J=32.2 \mathrm{~Hz}$ ), 93.1, 101.9, 123.3 (q, $J=286.1 \mathrm{~Hz}$ ), 127.5, 127.8, 129.0; IR (neat, $\mathrm{cm}^{-1}$ ) 2957, 2878, 2170, 1459, 1415, 1266, 1184, 846, 846, 742, 698; EI-HRMS Calcd for $\mathrm{C}_{22} \mathrm{H}_{35} \mathrm{~F}_{3} \mathrm{OSi}_{2}{ }^{+}\left(\mathrm{M}^{+}\right) 428.2173$, Found 428.2168.

5-Trifluoromethyl-1,7-diphenylhept-3-yn-5-ol (5bb): ${ }^{1} \mathrm{H}$ NMR (500 $\left.\mathrm{MHz}, \mathrm{CDCl}_{3}\right) \delta$ HO $\mathrm{CF}_{3} \quad 1.88-2.03(\mathrm{~m}, 2 \mathrm{H}), 2.39(\mathrm{~s}, 1 \mathrm{H}), 2.49(\mathrm{t}, J=7.4 \mathrm{~Hz}, 2 \mathrm{H}), 2.71-2.80$ $\mathrm{Ph}$ $(\mathrm{m}, 4 \mathrm{H}), 7.08-7.16(\mathrm{~m}, 6 \mathrm{H}), 7.19-7.24(\mathrm{~m}, 4 \mathrm{H}) ;{ }^{19} \mathrm{~F} \mathrm{NMR}(466 \mathrm{MHz}$, $\left.\mathrm{CDCl}_{3}\right) \delta-81.7 ;{ }^{13} \mathrm{C} \mathrm{NMR}\left(125 \mathrm{MHz} \mathrm{CDCl}_{3}\right) \delta 20.7,29.6,34.4$, $36.5,71.6$ (q, $J=31.4 \mathrm{~Hz}$ ), 75.9, 88.3, 124.0 (q, $J=284.1 \mathrm{~Hz}), 126.1,126.5,128.4,128.5$, 139.9, 141.0; IR (neat, $\mathrm{cm}^{-1}$ ) 3530, 3443, 3029, 2937, 2245, 1176, 750, 700; EI-HRMS Calcd for $\mathrm{C}_{20} \mathrm{H}_{19} \mathrm{~F}_{3} \mathrm{O}^{+}\left(\mathrm{M}^{+}\right) 332.1383$, Found 332.1384.

1-Triethylsilyl-3-trifluoromethyl-5-phenylpent-1-yn-3-ol (5bc): ${ }^{1} \mathrm{H} \quad \mathrm{NMR} \quad(500 \mathrm{MHz}$, HO $\left.\mathrm{CF}_{3} \quad \mathrm{CDCl}_{3}\right) \delta 0.58(\mathrm{q}, J=8.0 \mathrm{~Hz}, 6 \mathrm{H}), 0.95(\mathrm{t}, J=8.0 \mathrm{~Hz}, 9 \mathrm{H}), 1.96-2.09$ $\mathrm{SiEt}_{3} \quad(\mathrm{~m}, 2 \mathrm{H}), 2.50(\mathrm{~s}, 1 \mathrm{H}), 2.88(\mathrm{t}, J=8.6 \mathrm{~Hz}, 2 \mathrm{H}), 7.12-7.17(\mathrm{~m}, 3 \mathrm{H}), 7.23$ $(\mathrm{t}, J=8.0 \mathrm{~Hz}, 2 \mathrm{H}) ;{ }^{19} \mathrm{~F} \mathrm{NMR}\left(466 \mathrm{MHz}, \mathrm{CDCl}_{3}\right) \delta-81.6 ;{ }^{13} \mathrm{C} \mathrm{NMR}$ $\left(125 \mathrm{MHz}, \mathrm{CDCl}_{3}\right) \delta 3.8,7.3,29.8,36.6,72.0$ (q, $\left.J=31.4 \mathrm{~Hz}\right), 91.5,100.6,123.9(\mathrm{q}, J=$ $284.9 \mathrm{~Hz}$ ), 126.2, 128.4, 128.6, 141.0; IR (neat, $\mathrm{cm}^{-1}$ ) 3451, 3029, 2958, 2877, 2174, 1260, 1183, 1065, 729, 701; EI-HRMS Calcd for $\mathrm{C}_{18} \mathrm{H}_{25} \mathrm{~F}_{3} \mathrm{OSi}^{+}\left(\mathrm{M}^{+}\right)$342.1621, Found 342.1618 .

1-Triethylsilyl-3-triethylsilyloxy-3-trifluoromethyl-5-phenylpent-1-yn $\quad\left(\mathrm{Et}_{3} \mathrm{Si}-5 \mathrm{bc}\right): \quad{ }^{1} \mathrm{H}$ $\mathrm{Et}_{3} \mathrm{SiO} \quad \mathrm{CF}_{3} \quad \mathrm{NMR}\left(500 \mathrm{MHz}, \mathrm{CDCl}_{3}\right) \delta 0.64(\mathrm{q}, J=8.0 \mathrm{~Hz}, 6 \mathrm{H}), 0.73-0.79(\mathrm{~m}, 6 \mathrm{H})$, SiEt $_{3} 0.97(\mathrm{t}, J=8.6 \mathrm{~Hz}, 9 \mathrm{H}), 1.01(\mathrm{~d}, J=8.6 \mathrm{~Hz}, 9 \mathrm{H}), 1.53(\mathrm{~s}, 1 \mathrm{H})$, 2.01-2.13 (m, 2H), 2.78-2.90 (m, 2H), $7.18(\mathrm{~d}, J=7.6 \mathrm{~Hz}, 2 \mathrm{H}), 7.19$ (t, 
$J=7.6 \mathrm{~Hz}, 1 \mathrm{H}), 7.28(\mathrm{t}, J=7.6 \mathrm{~Hz}, 2 \mathrm{H}) ; 19 \mathrm{~F} \mathrm{NMR}\left(466 \mathrm{MHz}, \mathrm{CDCl}_{3}\right) \delta-80.2 ;{ }^{13} \mathrm{C} \mathrm{NMR}$ $\left(125 \mathrm{MHz}, \mathrm{CDCl}_{3}\right) \delta 4.0,5.8,6.8,7.3,30.1,40.1,72.8$ (q, $\left.J=31.0 \mathrm{~Hz}\right), 91.9,101.7,124.0$ (q, $J=279.0 \mathrm{~Hz}$ ), 126.1, 128.4 128.5, 141.0; IR (neat, $\left.\mathrm{cm}^{-1}\right)$ 2956, 2876, 2371, 1179, 1099, 739, 699; EI-HRMS Calcd for $\mathrm{C}_{24} \mathrm{H}_{39} \mathrm{~F}_{3} \mathrm{OSi}_{2}{ }^{+}\left(\mathrm{M}^{+}\right)$456.2486, Found 456.2489.

2-Cyclohexyl-1,1,1-trifluoro-4-phenylbut-3-yn-2-ol(5ka): ${ }^{1} \mathrm{H}$ NMR (500 $\left.\mathrm{MHz}, \mathrm{CDCl}_{3}\right) \delta$ HO $\mathrm{CF}_{3} \quad 1.12-1.45(\mathrm{~m}, 5 \mathrm{H}), 1.68-1.76(\mathrm{~m}, 1 \mathrm{H}), 1.79-1.90(\mathrm{~m}, 2 \mathrm{H}), 1.91-2.03(\mathrm{~m}$, $2 \mathrm{H}), 2.09-2.15(\mathrm{~m}, 1 \mathrm{H}), 2.67(\mathrm{~s}, 1 \mathrm{H}), 7.31-7.40(\mathrm{~m}, 3 \mathrm{H}), 7.48(\mathrm{~d}, J=8.0$ $\mathrm{Hz}, 2 \mathrm{H}) ;{ }^{19} \mathrm{~F} \mathrm{NMR}\left(466 \mathrm{MHz}, \mathrm{CDCl}_{3}\right) \delta-76.5 ;{ }^{13} \mathrm{C} \mathrm{NMR}(125 \mathrm{MHz}$, $\mathrm{CDCl}_{3}$ ) $\delta 25.9,26.0,26.8,27.40,27.42,43.1,75.1$ (q, $J=31.1 \mathrm{~Hz}$ ), 83.3, 87.7, 121.3, 124.4 (q, $J=288.4 \mathrm{~Hz}$ ), 128.4, 129.2, 132.0; IR (neat, $\mathrm{cm}^{-1}$ ) 3452, 3057, 2932, 2857, 2234, 1170, 756, 690; EI-HRMS Calcd for $\mathrm{C}_{16} \mathrm{H}_{17} \mathrm{~F}_{3} \mathrm{O}^{+}\left(\mathrm{M}^{+}\right)$282.1226, Found 282.1231. 
Table S1. Screening of Diphosphine Ligands for Racemic Catalytic Alkynylation of Trifluoroacetophenone (3a):

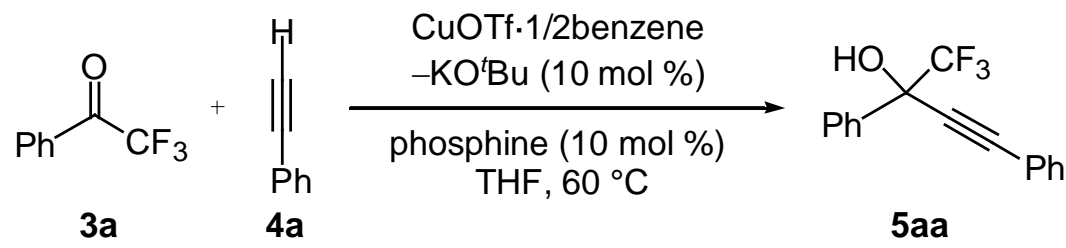

\begin{tabular}{cccc}
\hline entry & phosphine & time & yield \\
\hline 1 & dppe & 26 & 42 \\
2 & dppp & 26 & 53 \\
3 & dppf & 26 & 29 \\
4 & xantphos & 22 & $>99$
\end{tabular}<smiles>[Y]C1(C)c2cccc(P)c2Oc2c(P)cccc21</smiles>

Table S2. Screening of Diphosphine Ligands for Catalytic Asymmetric Alkynylation of Trifluoroacetophenone (3a):

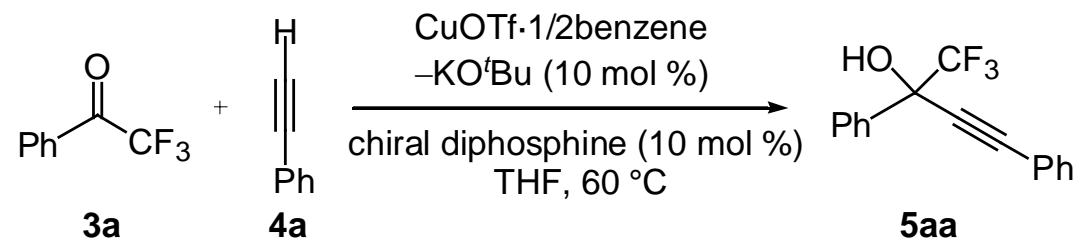

\begin{tabular}{ccccc}
\hline entry & diphosphine & time & yield & ee \\
\hline 1 & tol-BINAP & 16 & $>99$ & 37 \\
2 & H8-BINAP & 19 & 90 & 17 \\
3 & DM-BINAP & 14 & 75 & 39 \\
4 & BINAPO & 17 & NR & \\
5 & C3-TUNEPHOS & 23 & 94 & 40 \\
6 & DIFLUOROPHOS & 17 & 23 & 48 \\
7 & DTBM-SEGPHOS & 21 & $>99$ & 42 \\
8 & CTH-P-PHOS & 12 & 48 & 44 \\
9 & CTH-Xy2-P-PHOS & 12 & $>99$ & 41 \\
10 & iPr-DuPHOS & 17 & 95 & 6 \\
11 & JOSIPHOS & 23 & $>99$ & 11 \\
12 & SL-M001-1 & 23 & 59 & 13 \\
13 & SL-M002-1 & 23 & $>99$ & 22 \\
14 & SL-TO02-1 & 17 & $>99$ & 6 \\
15 & BICP & 14 & 15 & 12 \\
16 & TANGPHOS & 14 & 85 & 10 \\
17 & NORPHOS & 19 & trace &
\end{tabular}


Table S3. Screening of Amine Ligands for Catalytic Asymmetric Alkynylation of Trifluoroacetophenone (3a):

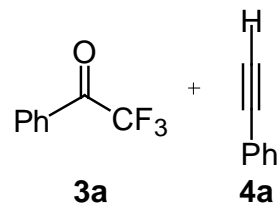

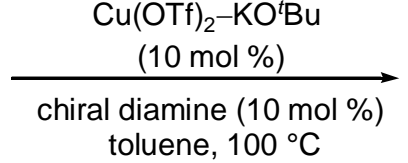
toluene, $100{ }^{\circ} \mathrm{C}$<smiles>OC(C#CPc1ccccc1)(c1ccccc1)C(F)(F)F</smiles>

5 aa<smiles>[R1]C1N=C(c2cccc(C3=N[C@@H]([R1])[C@@H]([R1])O3)n2)OC1[R1]</smiles>

$\mathrm{R}_{1}=\mathrm{Ph}: \mathrm{y} \cdot \%, 0-45 \%$ ee (not reproducible) $\mathrm{R}_{1}=$ 2-Naphthyl: No Rxn<smiles>c1ccc(C2N=C(c3cccc(-c4cccc(C5=N[C@@H](c6ccccc6)[C@H](c6ccccc6)O5)n4)n3)OC2c2ccccc2)cc1</smiles><smiles>c1ccc(C2N=C(c3ccc4ccccc4n3)OC2c2ccccc2)cc1</smiles>

y. $23 \%, 0 \%$ ee<smiles>C=CC1CC2CN1CC2[C@H](N=CNC)c1ccnc2ccccc12</smiles>

y. $37 \%, 1 \%$ ee

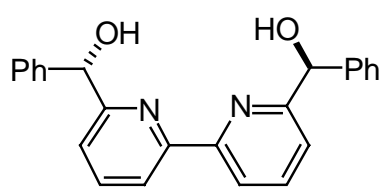

y. $6 \%, 1 \%$ ee<smiles>O=C(N[C@H](c1ccccc1)C(c1ccccc1)c1ccccc1)c1ccccc1</smiles>

No Rxn<smiles>c1ccc(CN[C@@H](c2ccccc2)[C@H](NCc2ccccc2)c2ccccc2)cc1</smiles>

$17 \%, 0 \%$ ee<smiles>C(=N/C(c1ccccc1)[C@H](/N=C/c1ccccc1)c1ccccc1)\c1ccccc1</smiles>

$16 \%, 0 \%$ ee

\section{Determination of Absolute Configuration of 5aa}

1) Red-Al, $\mathrm{Et}_{2} \mathrm{O}, 0^{\circ} \mathrm{C}, 1 \mathrm{~h}$

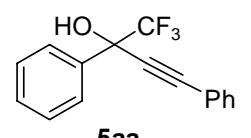

5 aa

3) $\mathrm{NaBH}_{4}, 1 \mathrm{~h}, 68 \%$ (3 steps)<smiles>OCC(O)(c1ccccc1)c1ccccc1</smiles>

$[\alpha]^{25}=+5.1(\mathrm{c}=1.29, \mathrm{MeOH})$

$[\alpha]_{D}=+17.2(\mathrm{c}=0.36, \mathrm{MeOH})$ for $\mathrm{S}$ isomer $^{4}$

$31 \%$ ee
using pybox 10

To a solution of $5 \mathrm{aa}(60 \mathrm{mg}, 0.22 \mathrm{mmol})$ in ether $(5 \mathrm{~mL})$, Red-Al (3M in toluene, $0.22 \mathrm{~mL}$, $0.65 \mathrm{mmol}$ ) was added at $0{ }^{\circ} \mathrm{C}$, and the mixture was stirred for $1 \mathrm{~h} .1 \mathrm{M} \mathrm{HCl}$ was added, and the products were extracted with AcOEt. Combined organic layers were washed with brine, and dried over $\mathrm{Na}_{2} \mathrm{SO}_{4}$. Filtration and evaporation of the solvent afforded the corresponding allylic alcohol, which was subjected to standard ozonolysis conditions at $-78{ }^{\circ} \mathrm{C}$. Addition of $\mathrm{NaBH}_{4}$ afforded the diol in $68 \%$ yield (3 steps). 4

1 Singh, R. P.; Cao, G.; Kirchmeier, R. L, Shreeve, J. M. J. Org. Chem. 1999, 64, $2873-2876$. 
2 Evans, D. A.; Song, H.-J.; Fandrick, K. R. Org. Lett. 2006, 8, 3351-3354.

${ }^{3}$ Linderman, R. J.; Lonikar, M. J. Org. Chem. 1988, 53, 6013-6022.

${ }_{4}^{4}$ Moreno-Dorado, F. J.; Guerra, F. M.; Ortega, M. J.; Zubia, E.; Massanet, G. M.

Tetrahedron: Asymmetry 2003, 14, 503-510. 

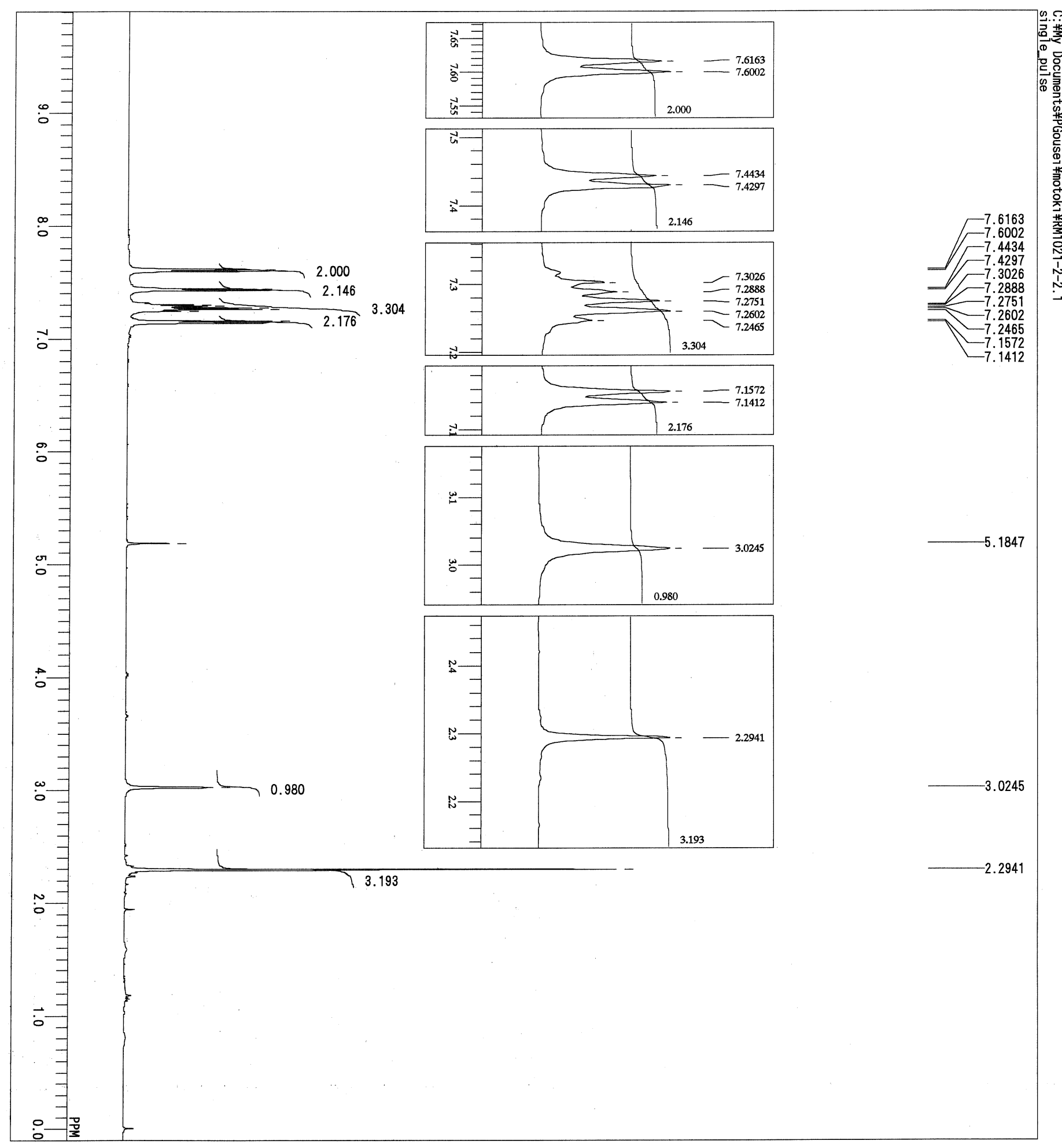

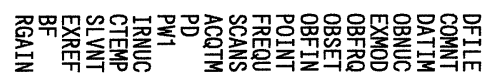

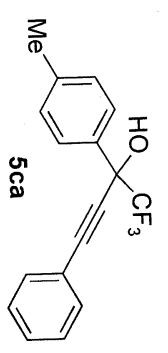

용포

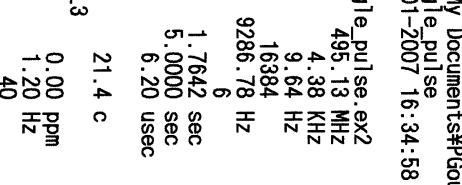




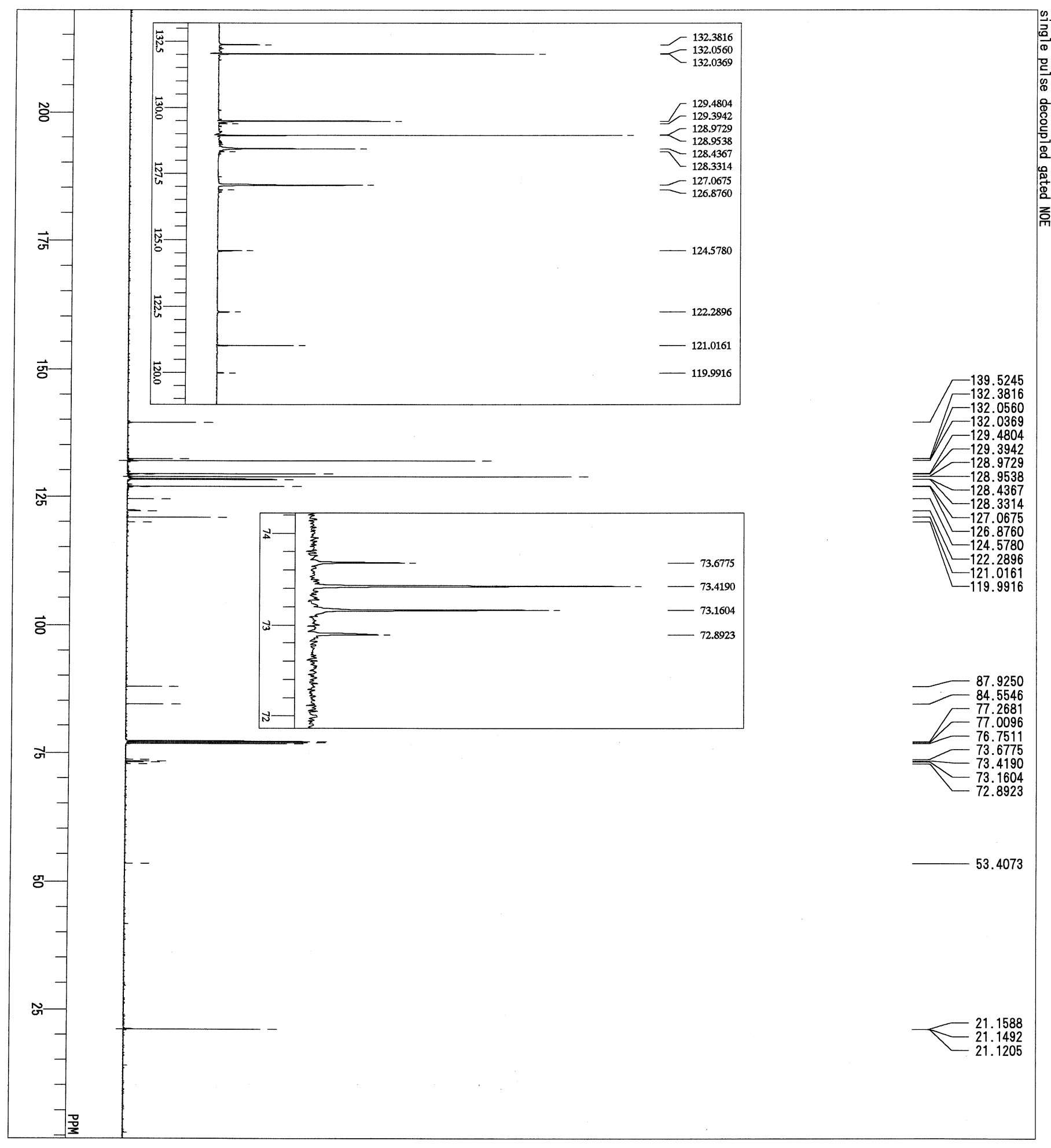

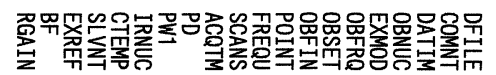

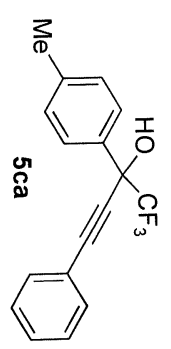



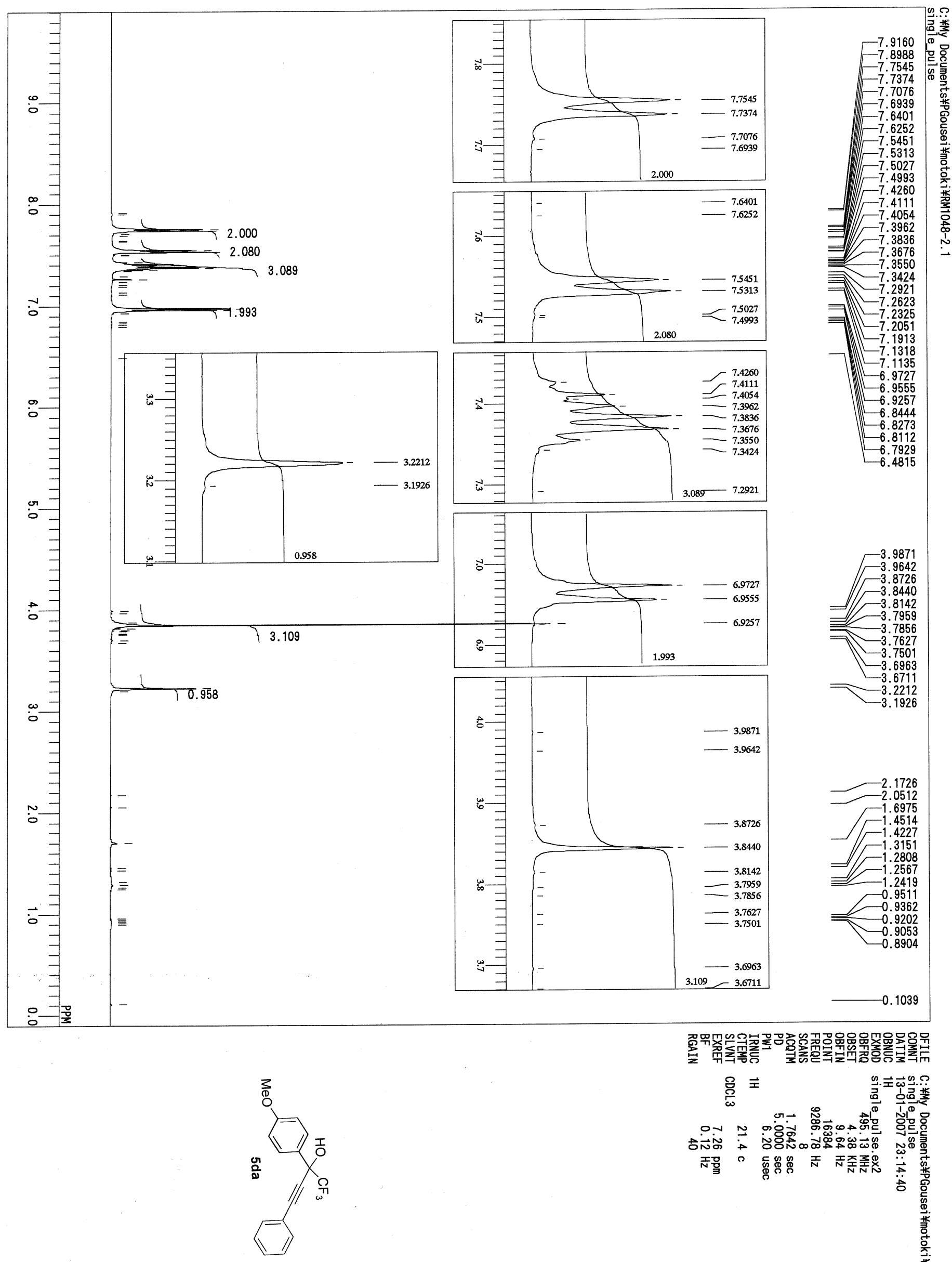

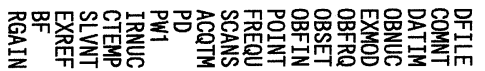

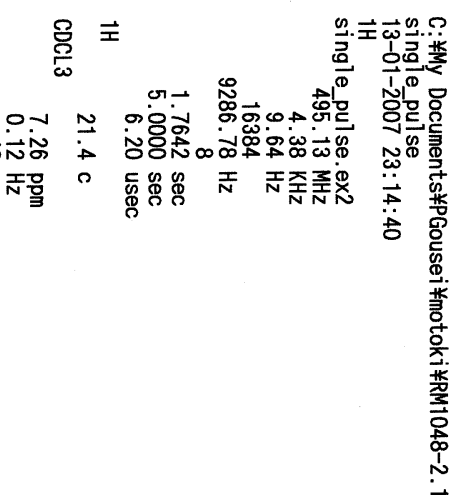




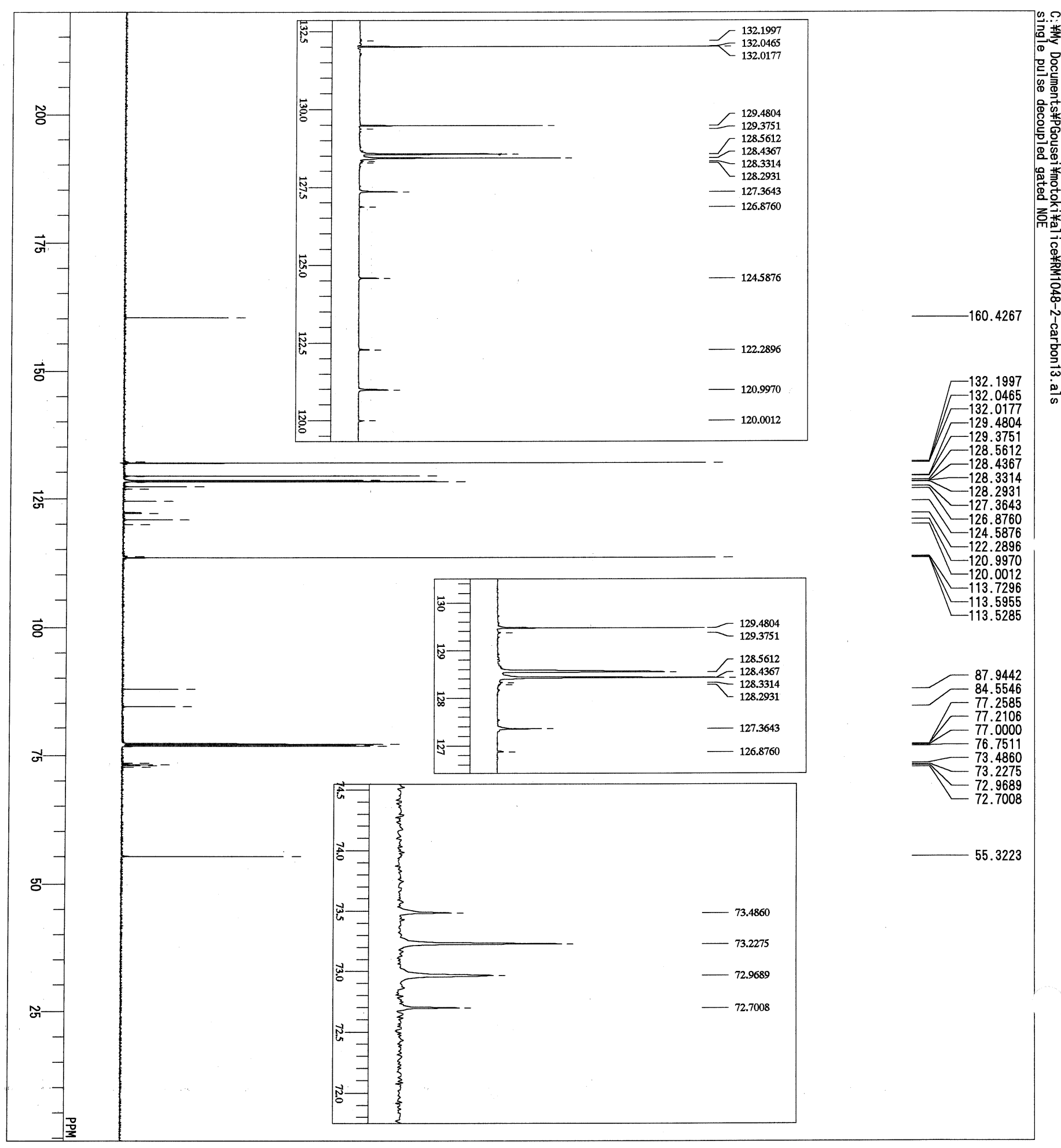

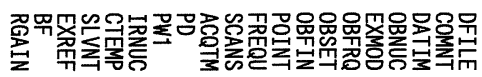

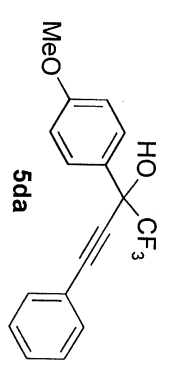

$\stackrel{8}{\frac{8}{\omega}} \vec{x}$

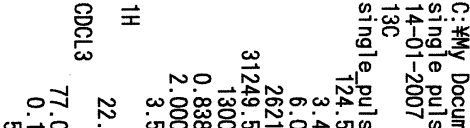
๓N

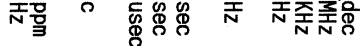



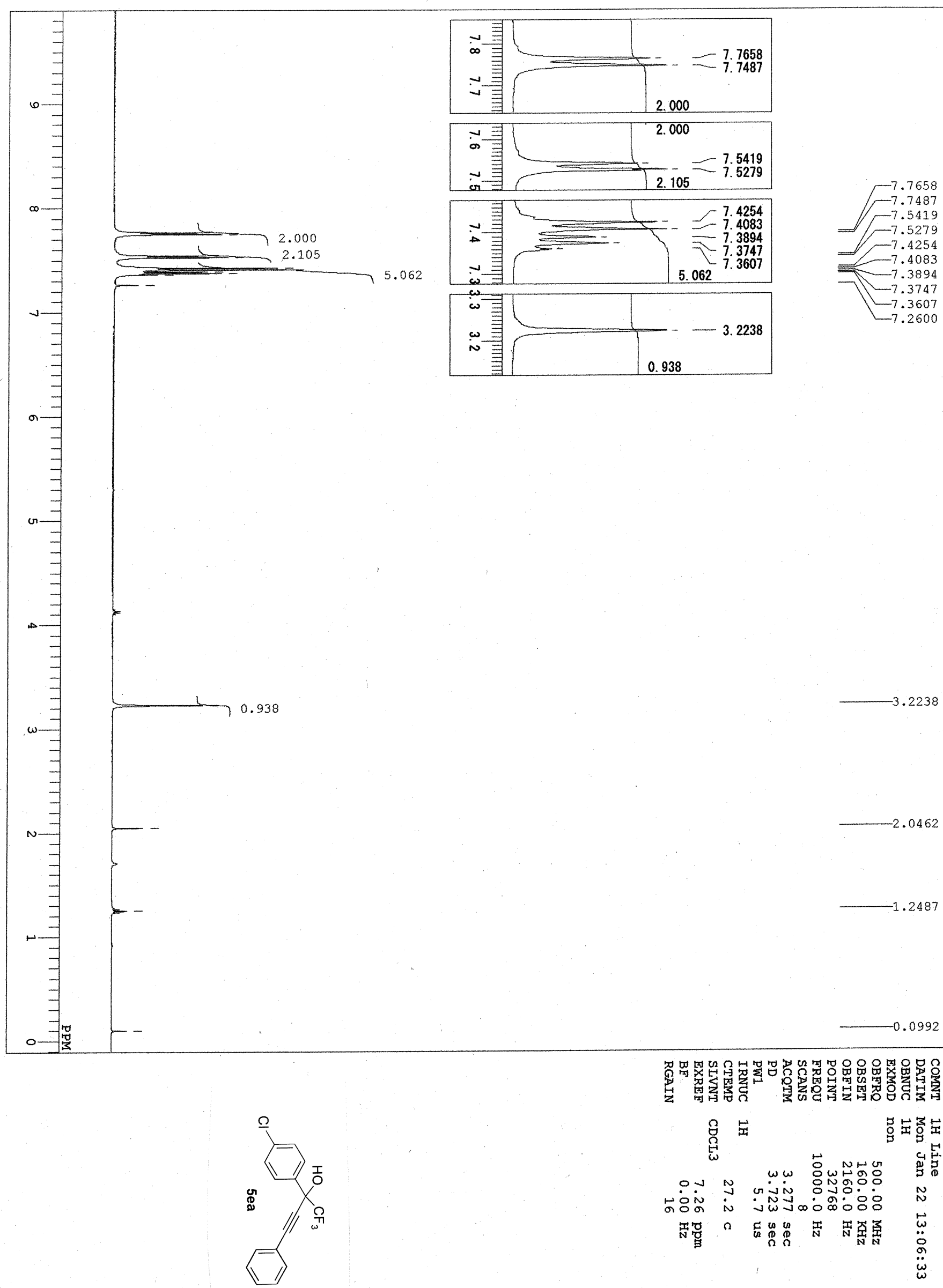


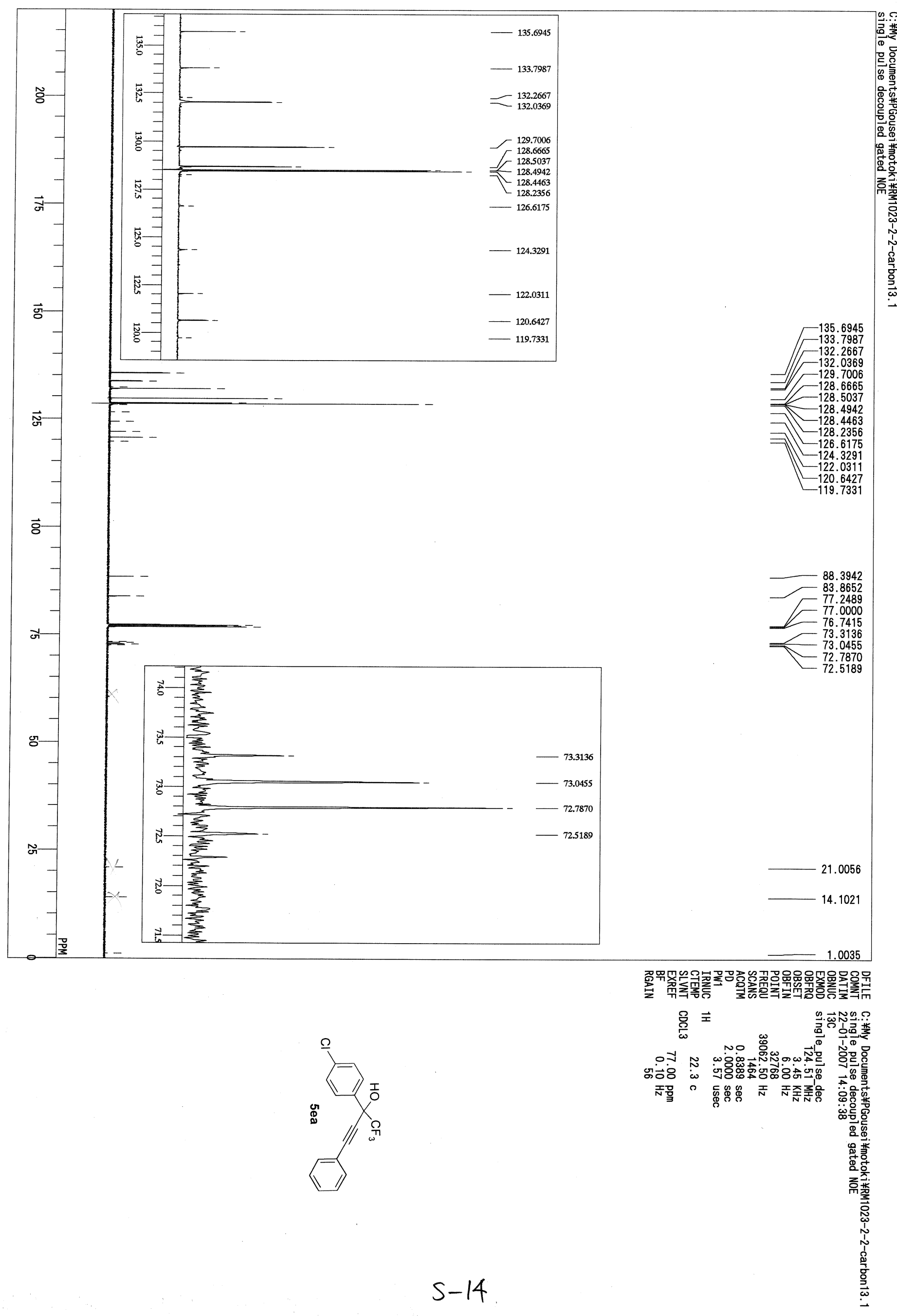




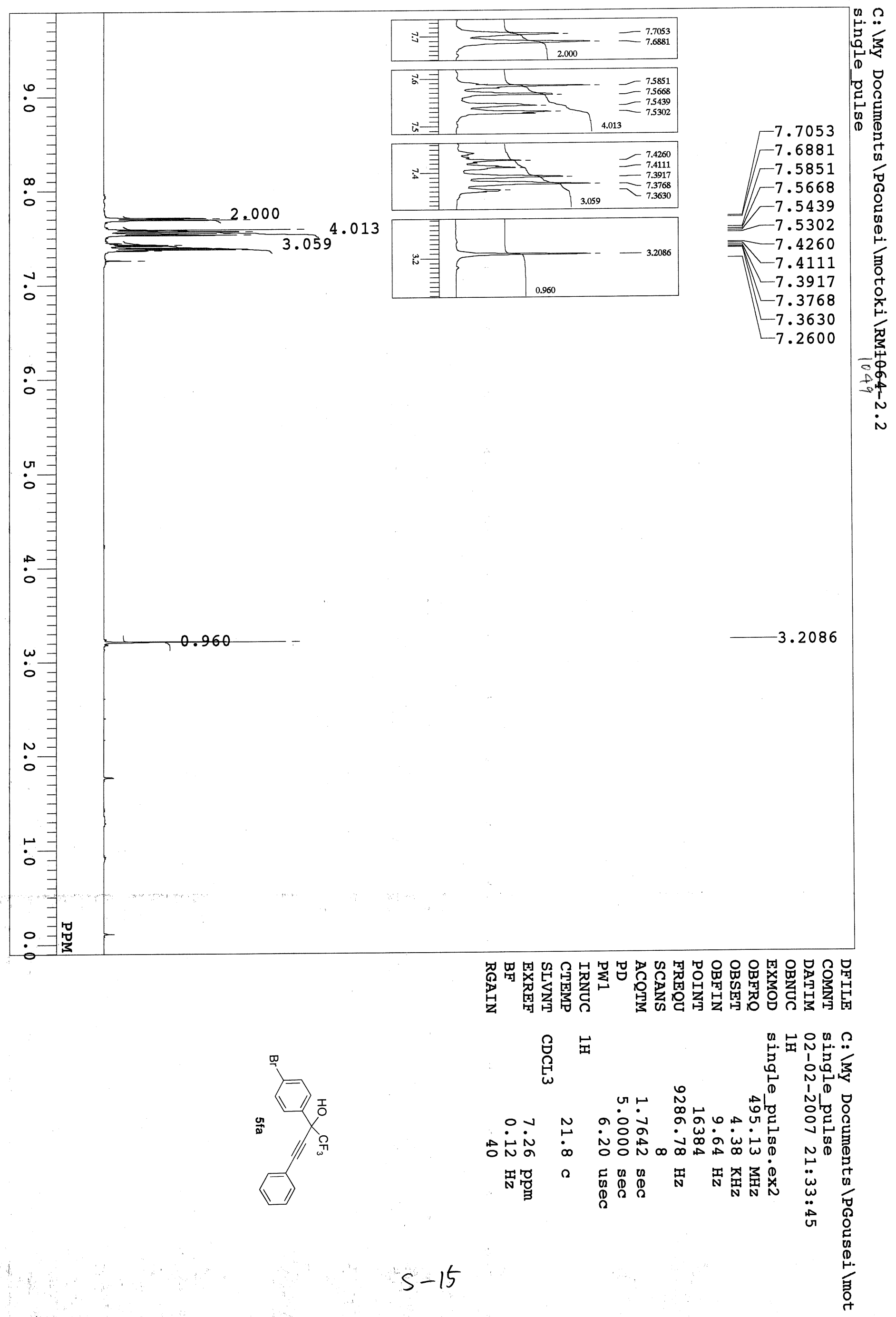




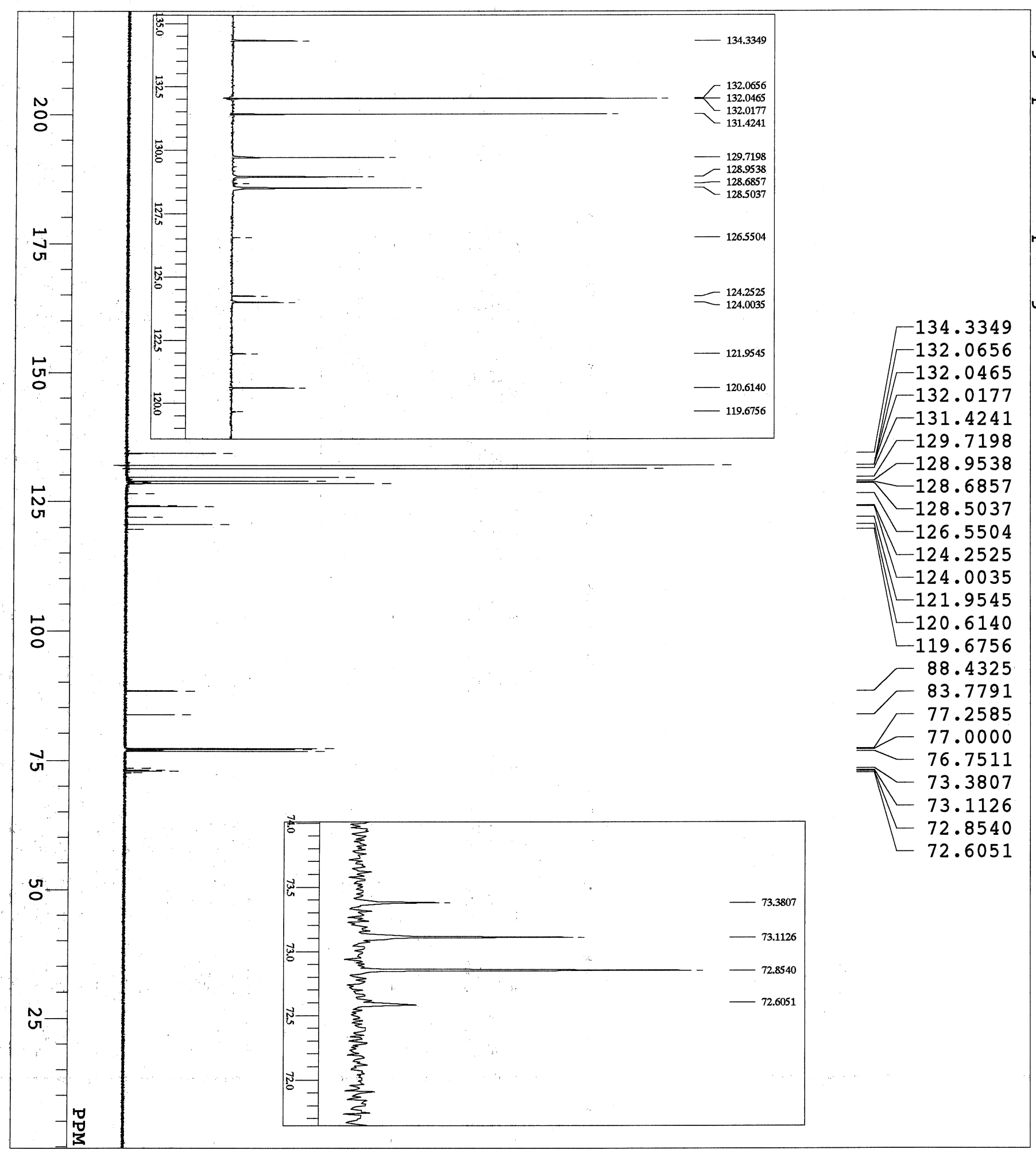

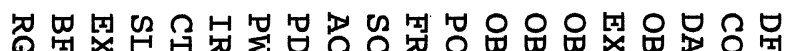

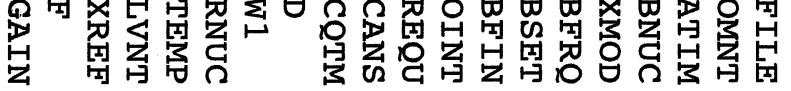

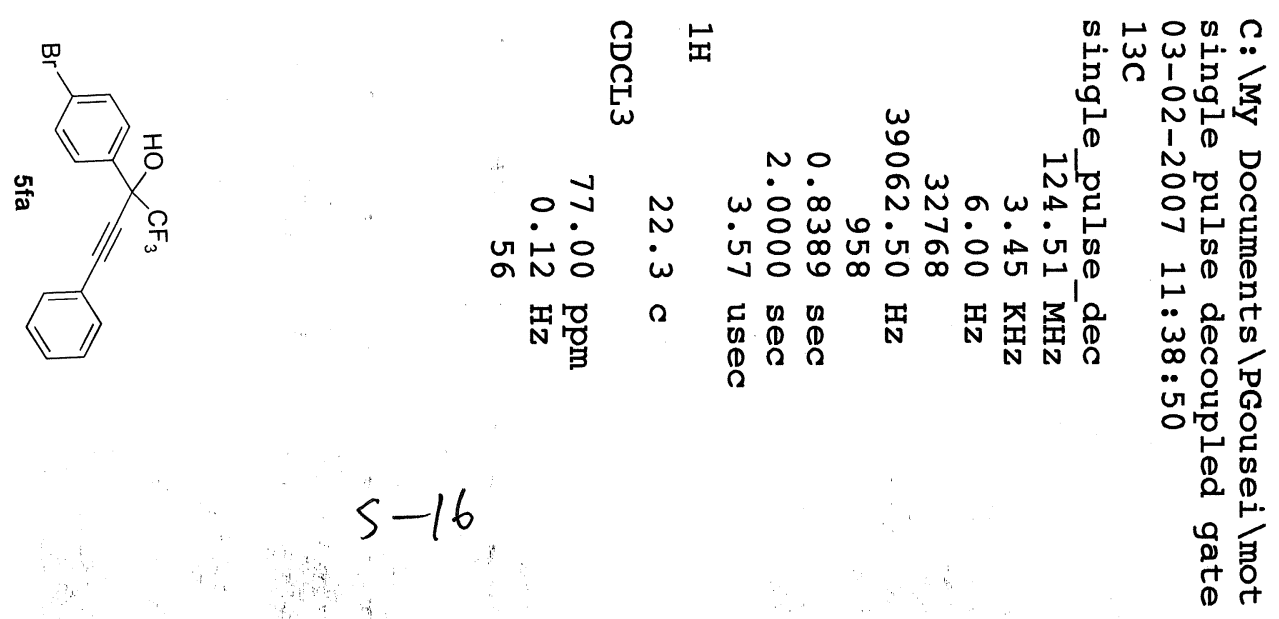




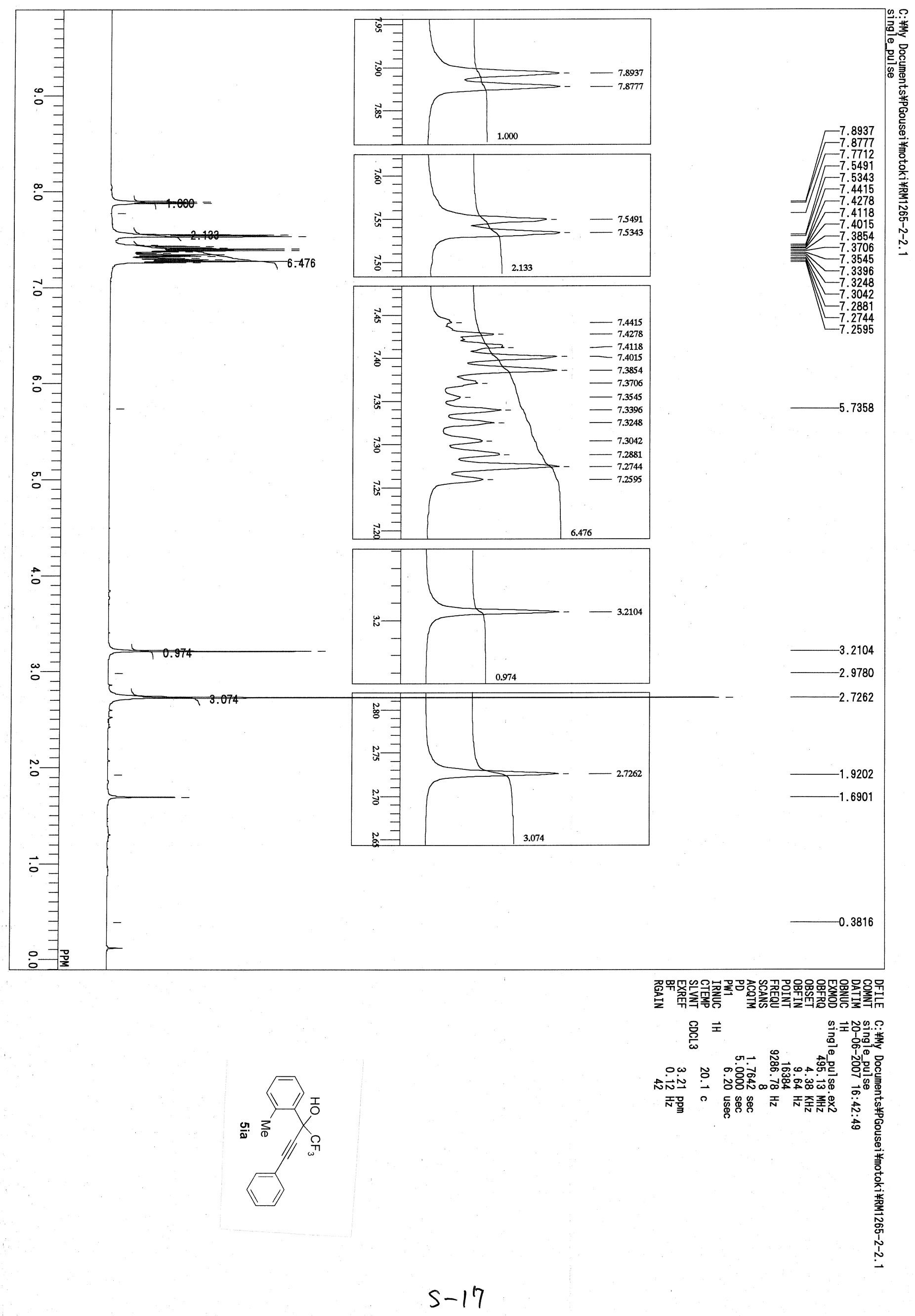




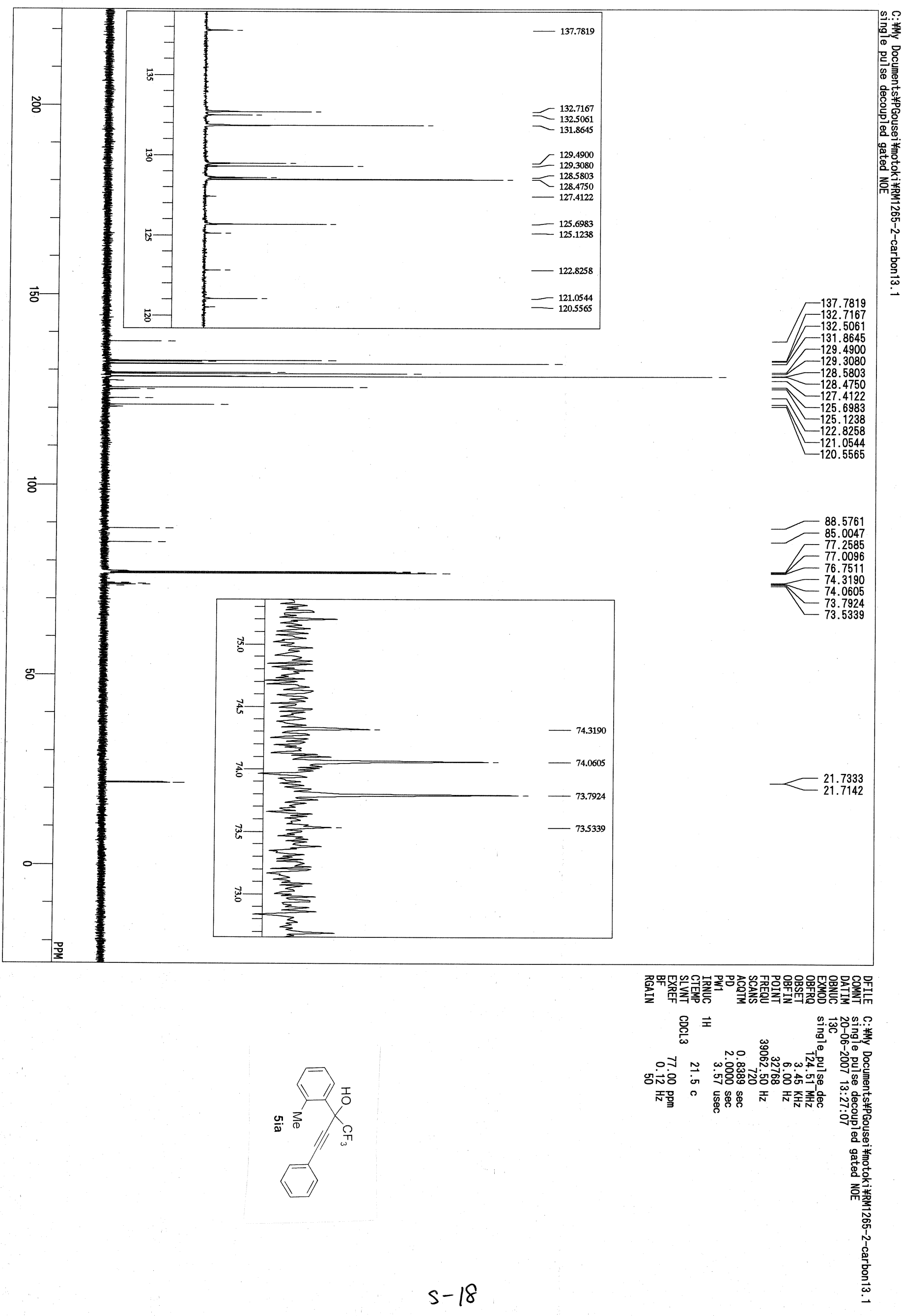








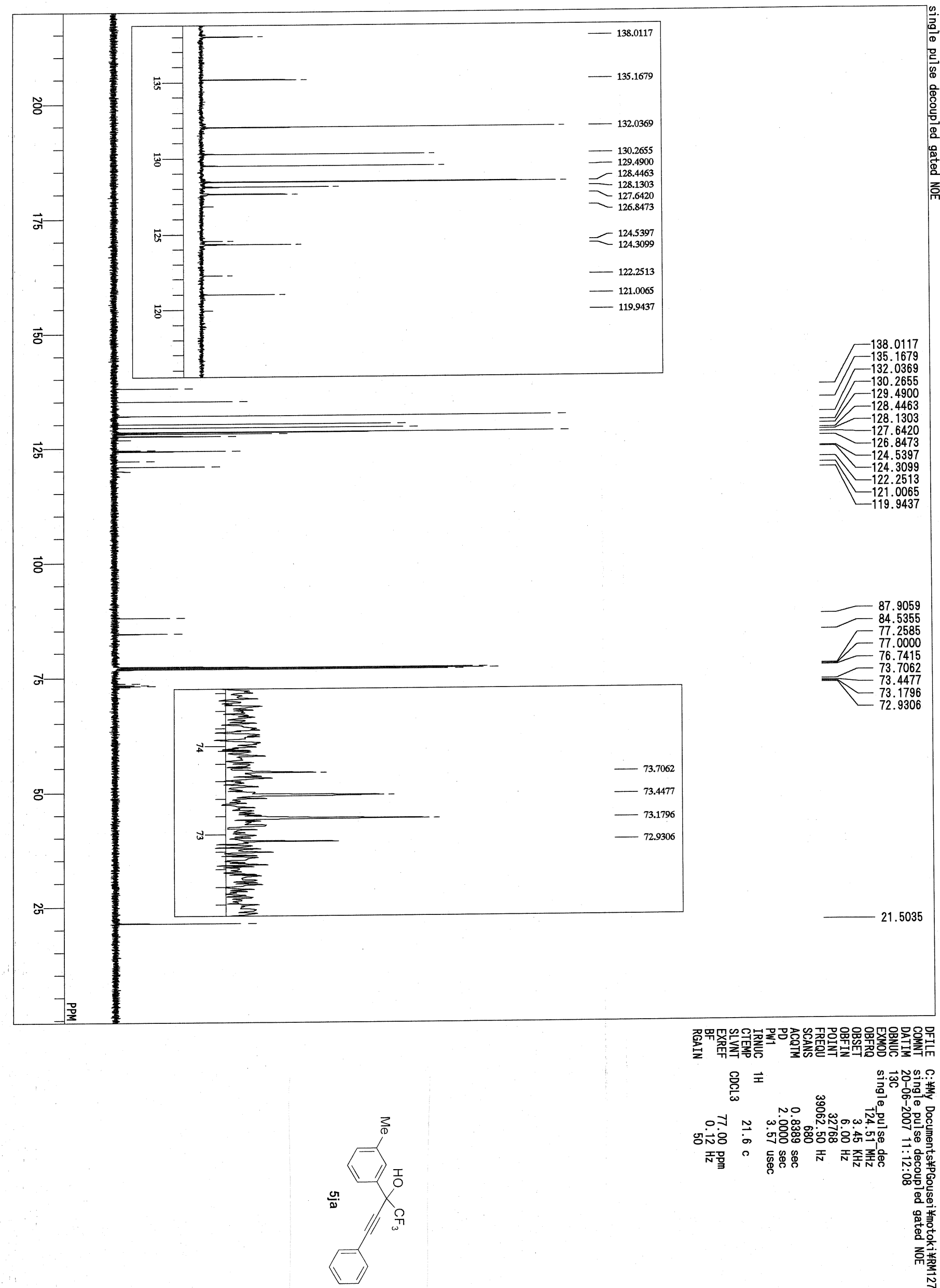
ㄱ No 䓵

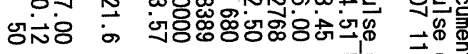

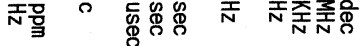




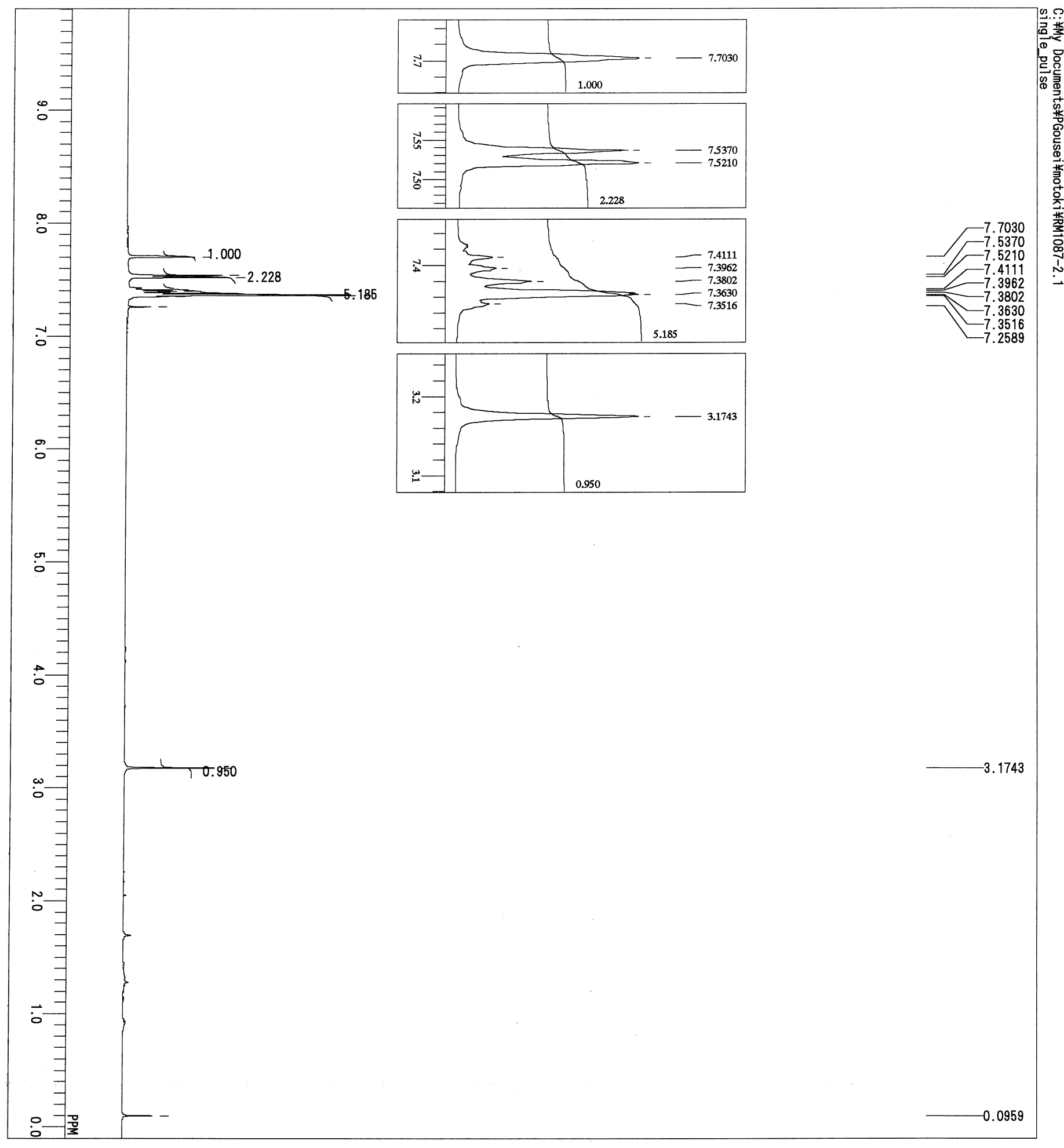

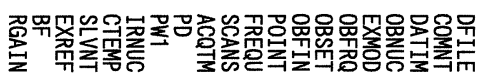
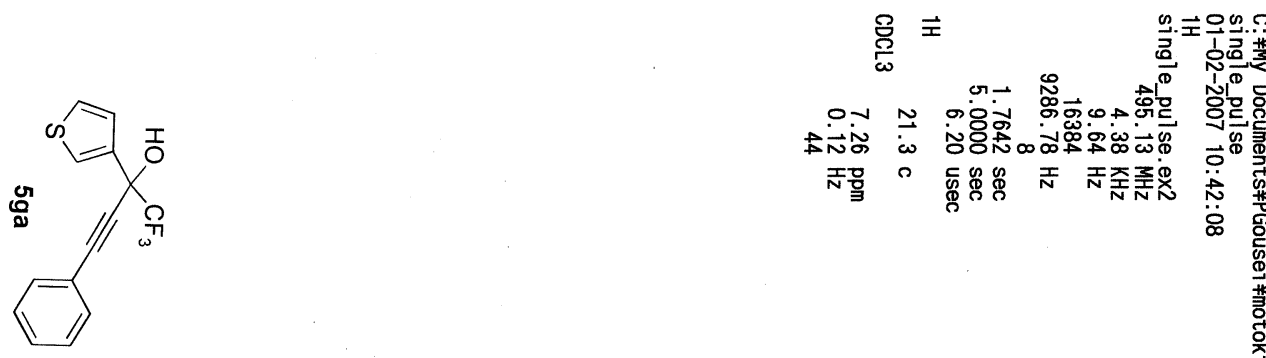


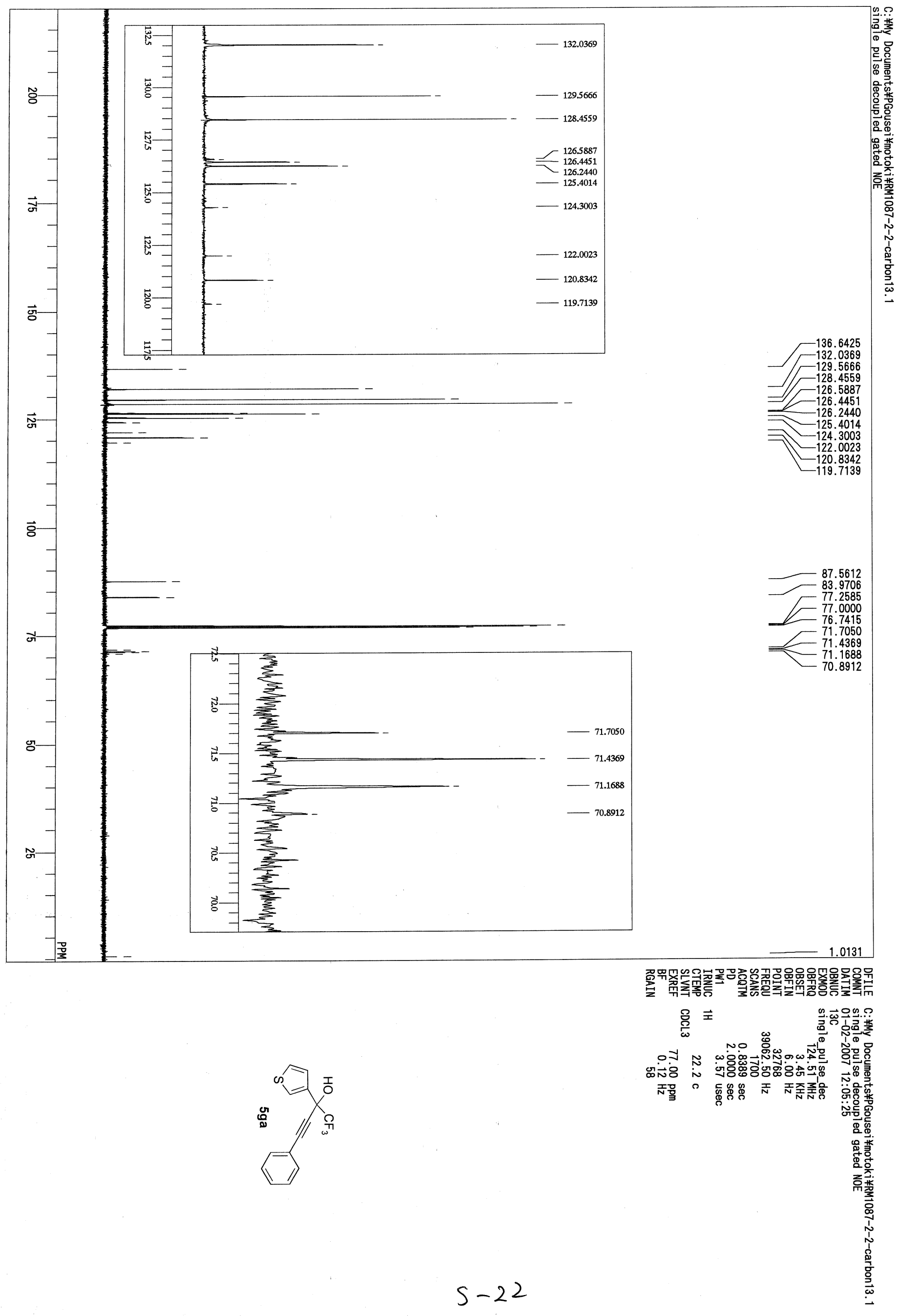




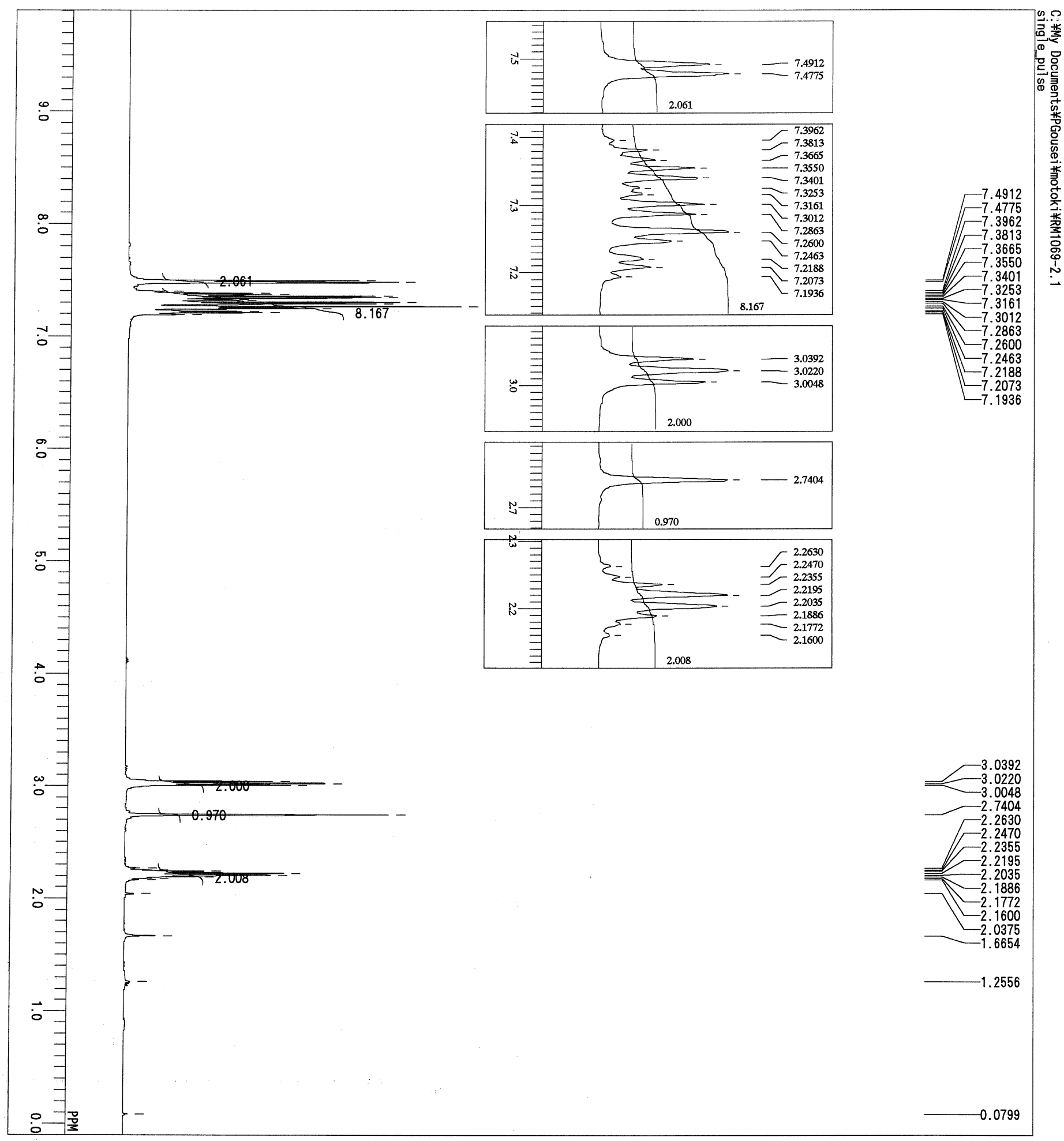

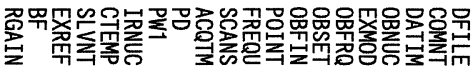
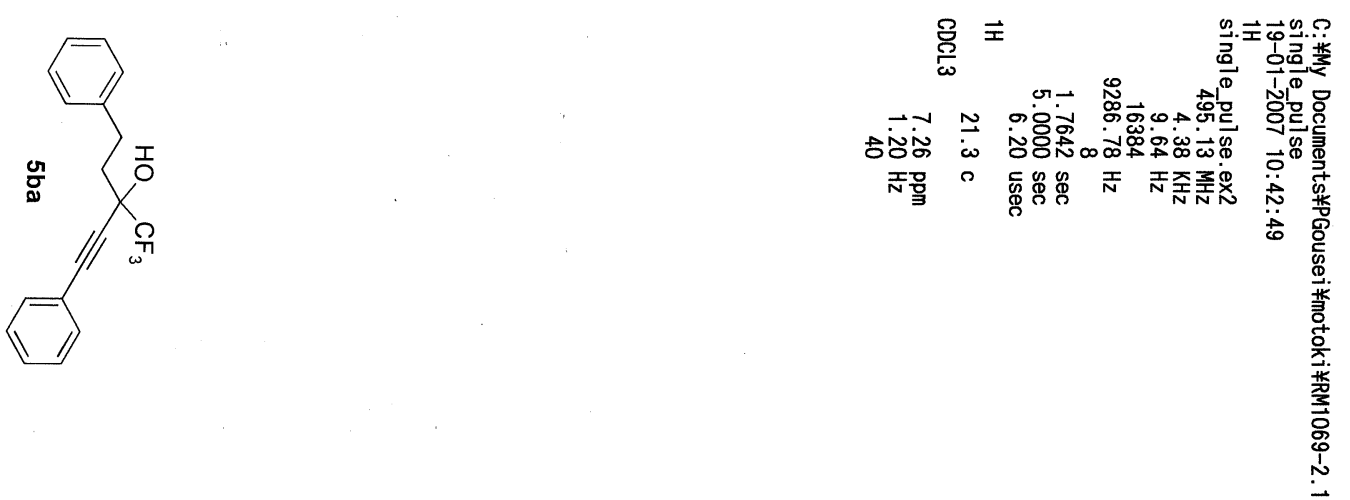

$S-23$ 


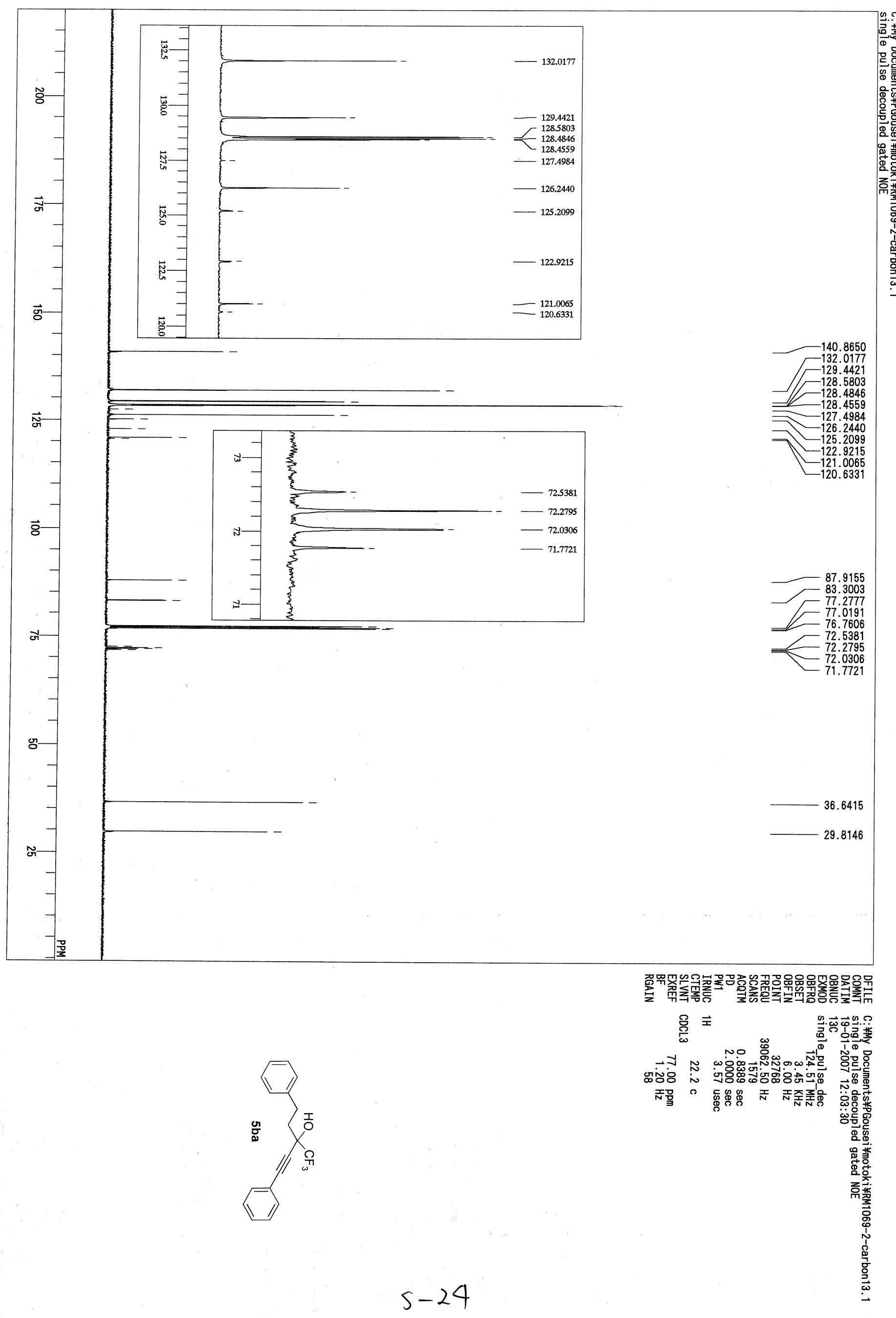




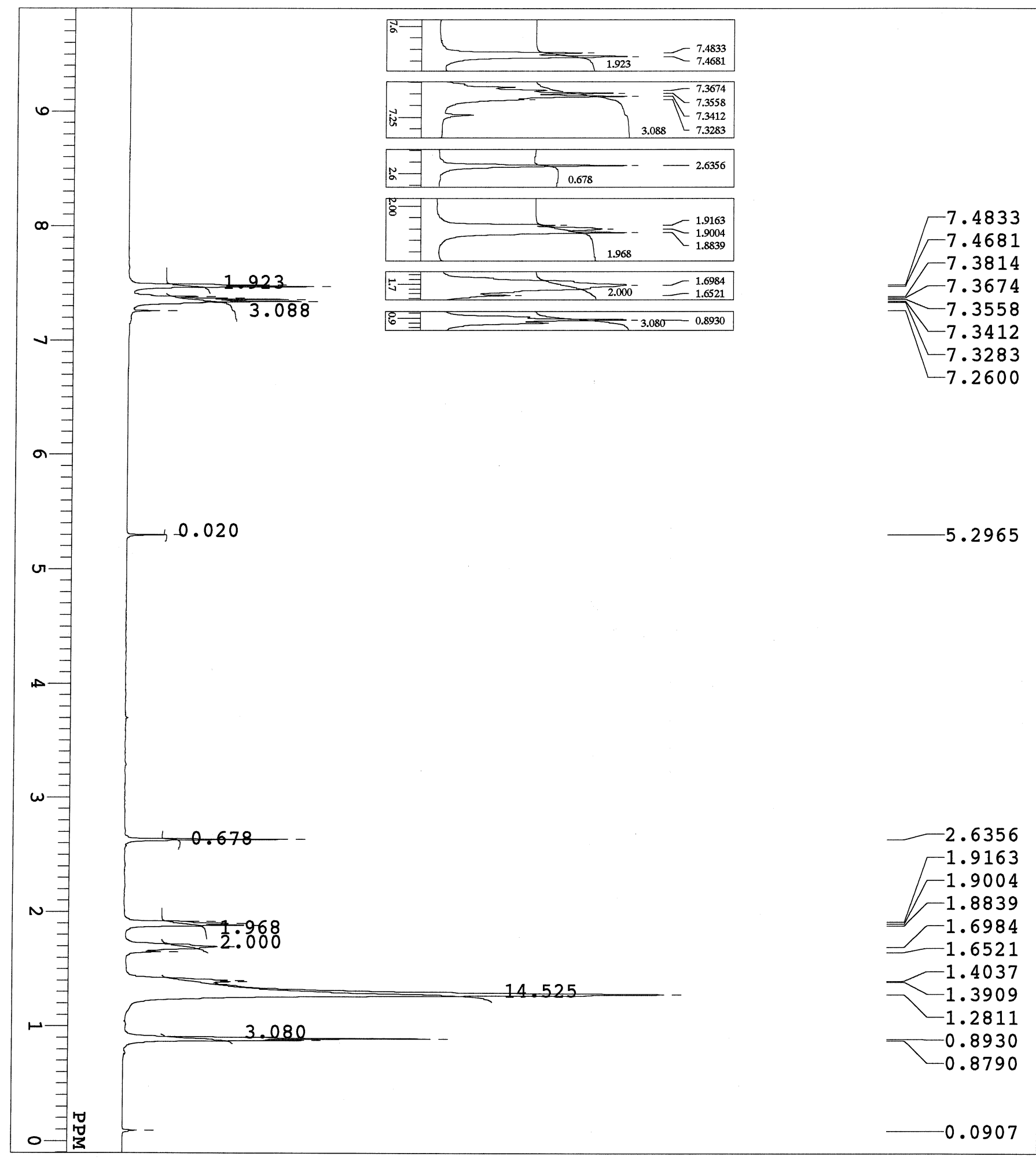

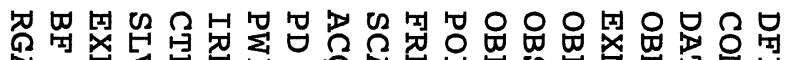

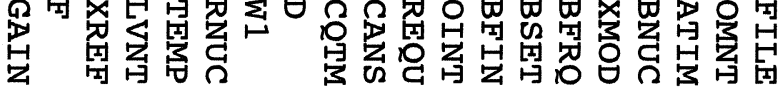

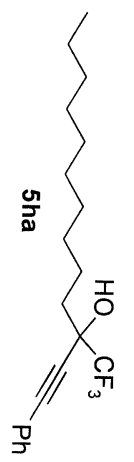

含 出

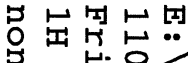

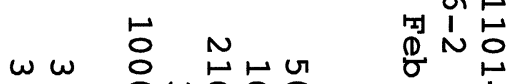

レ N in NNO

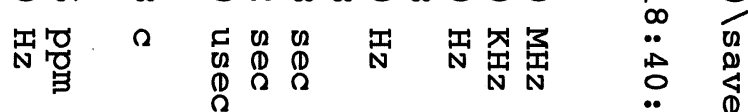
$\underset{\sim}{\ddot{\omega}} \quad \stackrel{0}{0}$ 

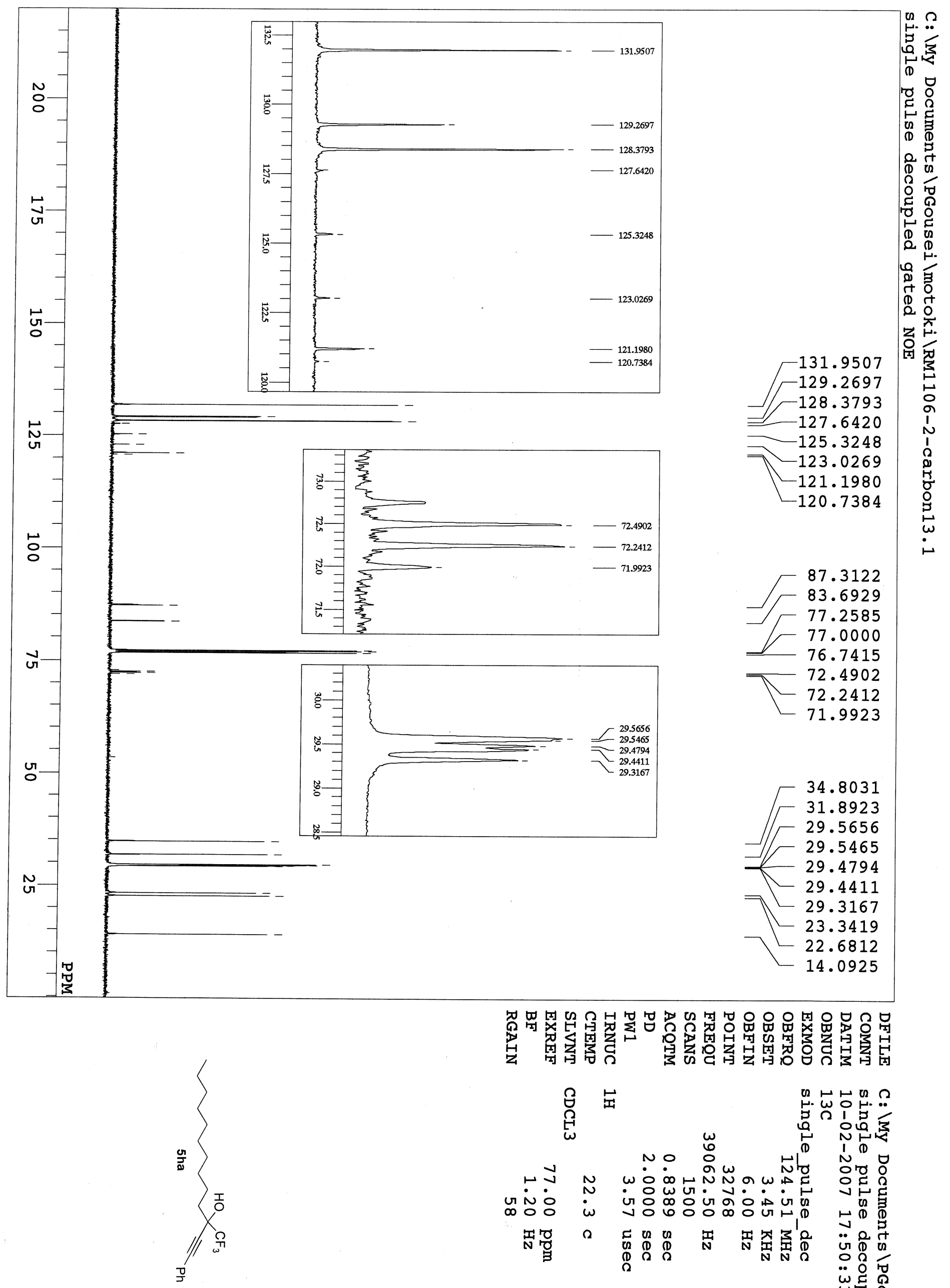

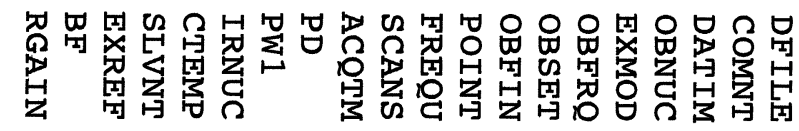

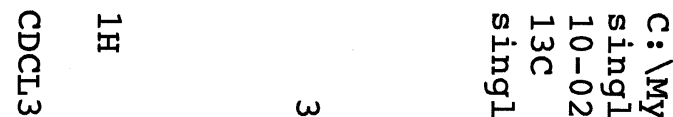

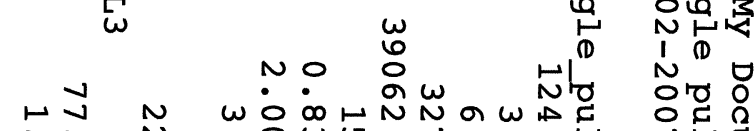
ज决 N N

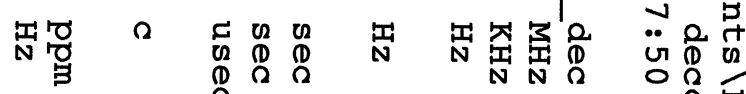
$\because 0$ 西 $\omega \mathscr{\omega}$ to 


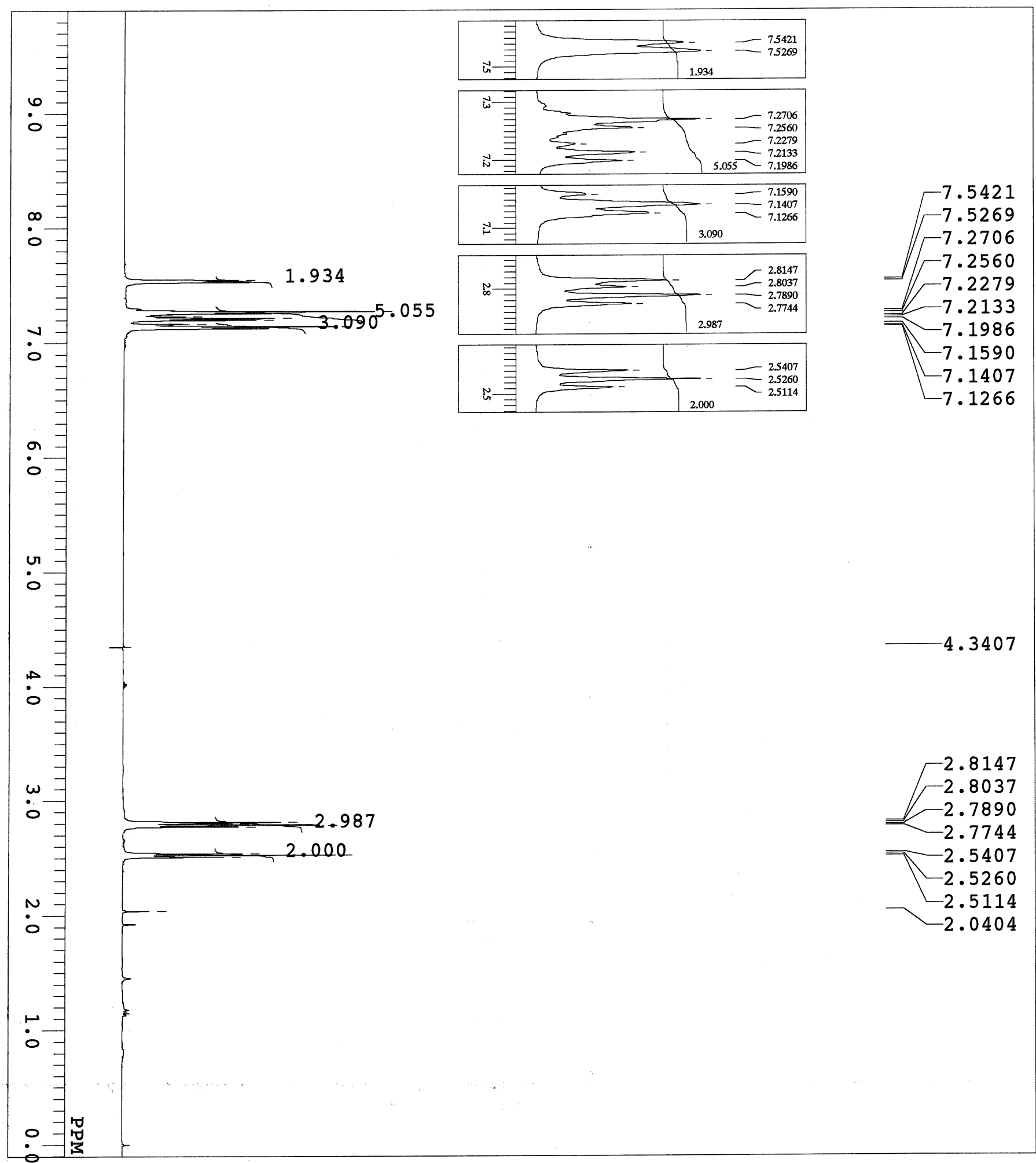

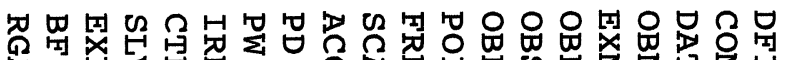

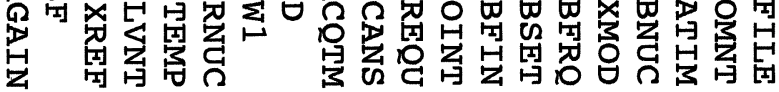

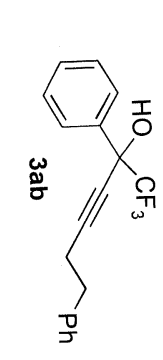<smiles>[Hg]=[Hg]</smiles>

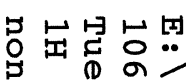
W o $N$ vin : $\begin{aligned} & \omega \omega \\ & \text { in }\end{aligned}$ मं० WN

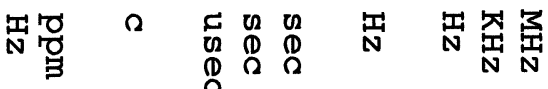




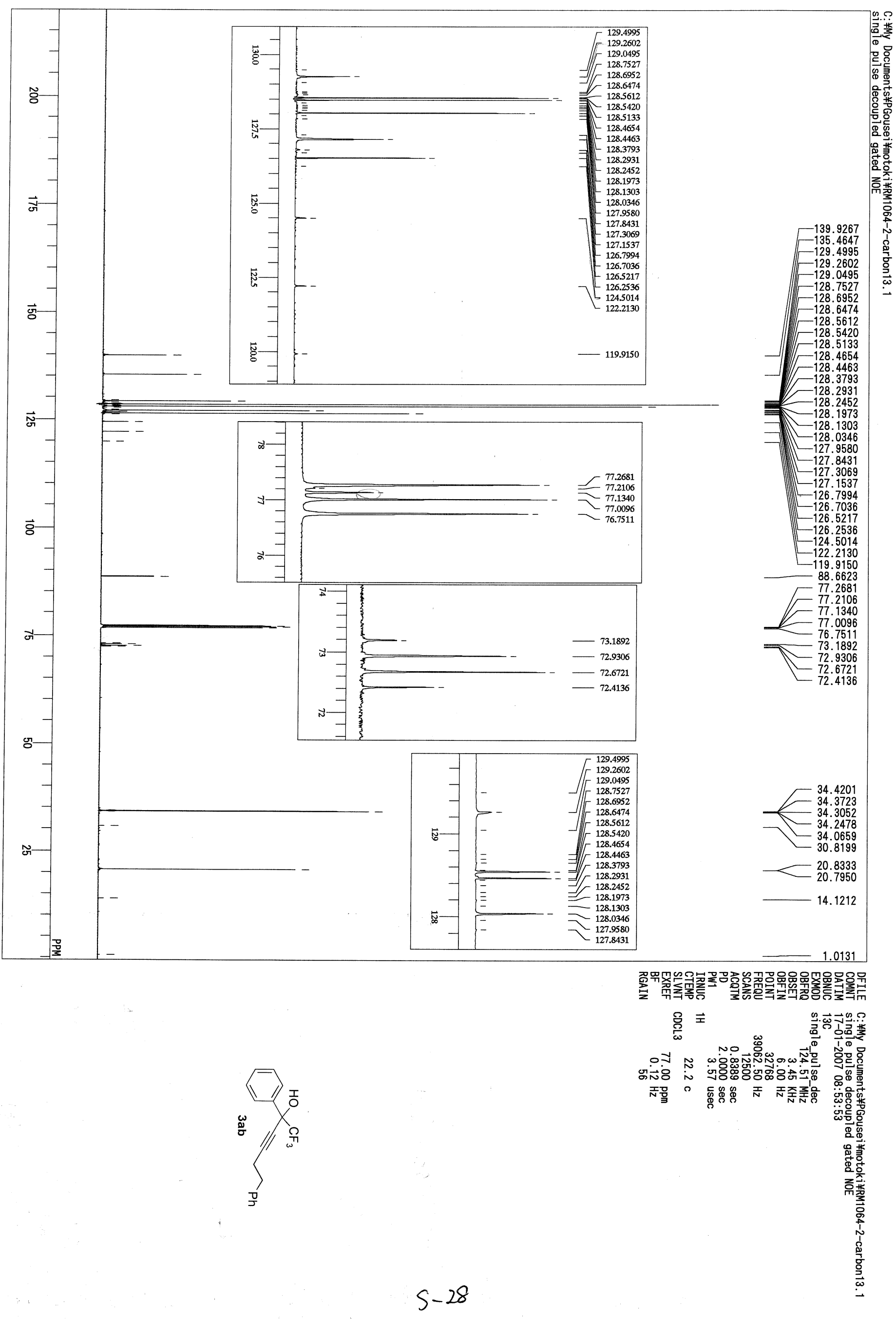




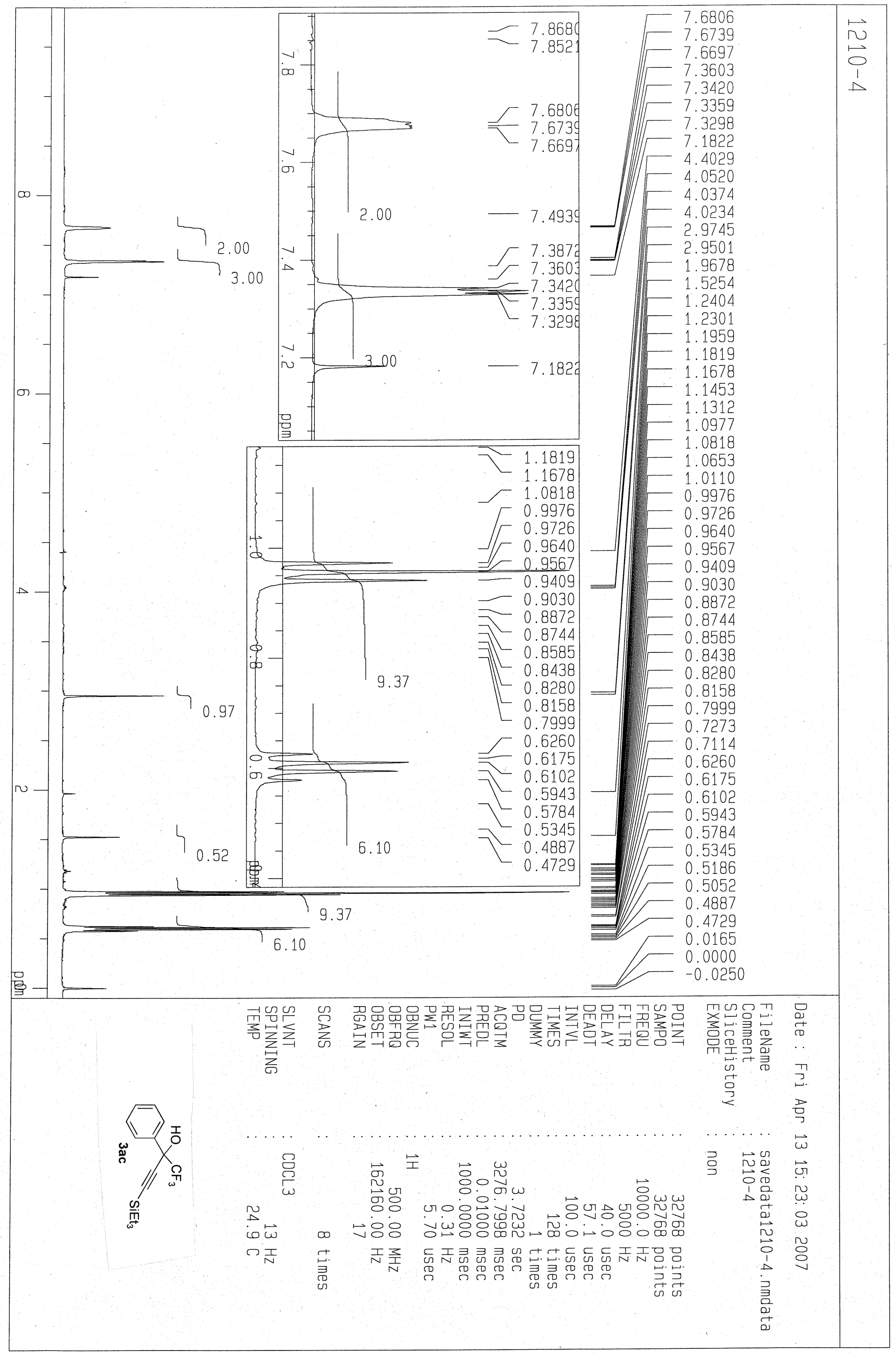




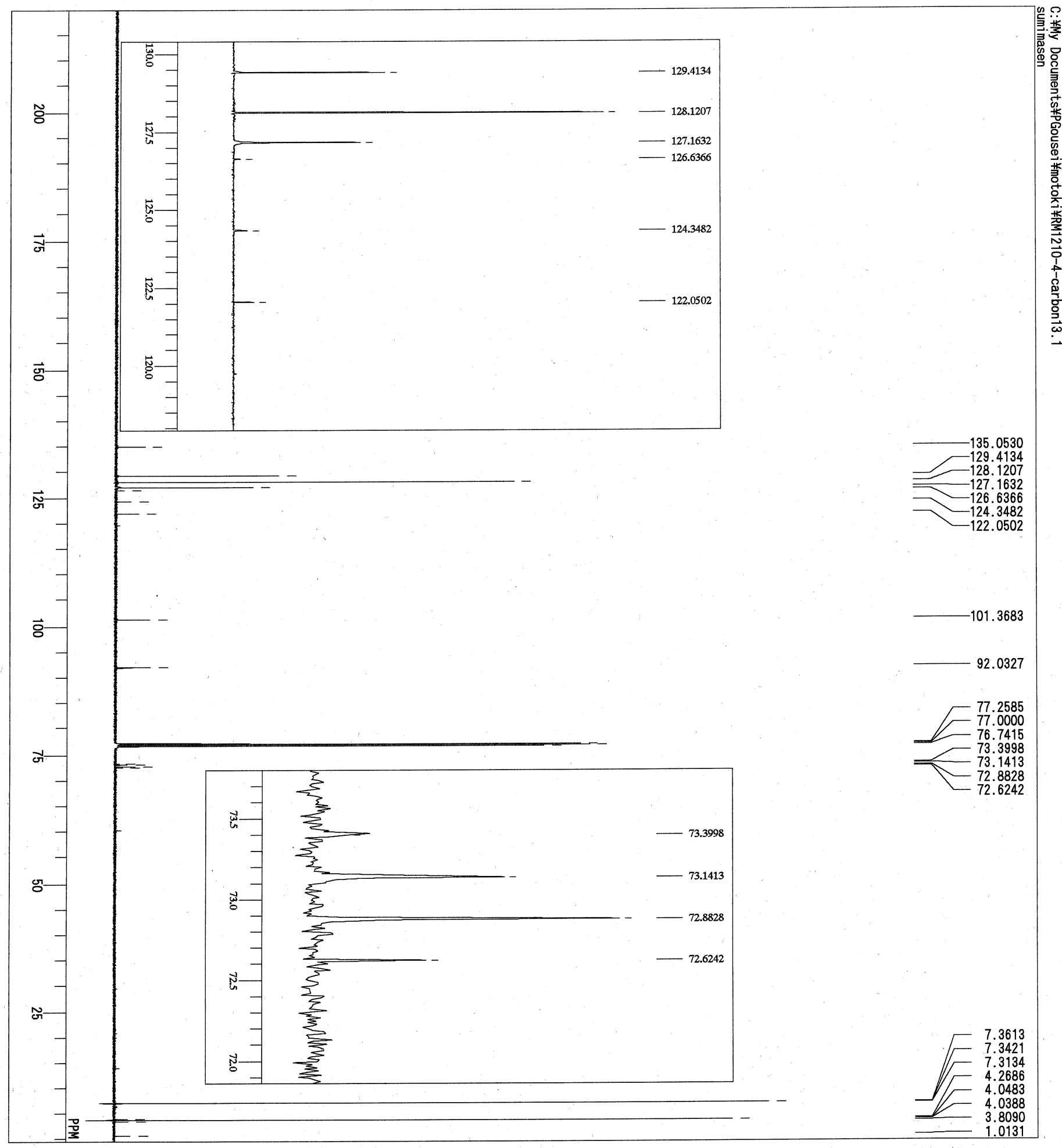

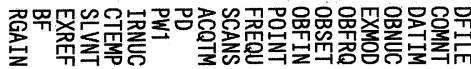

$\underset{\infty}{\infty}$

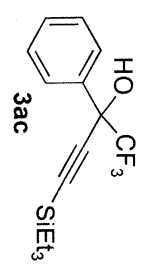

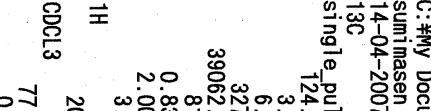

ज唍

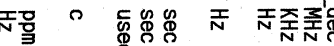



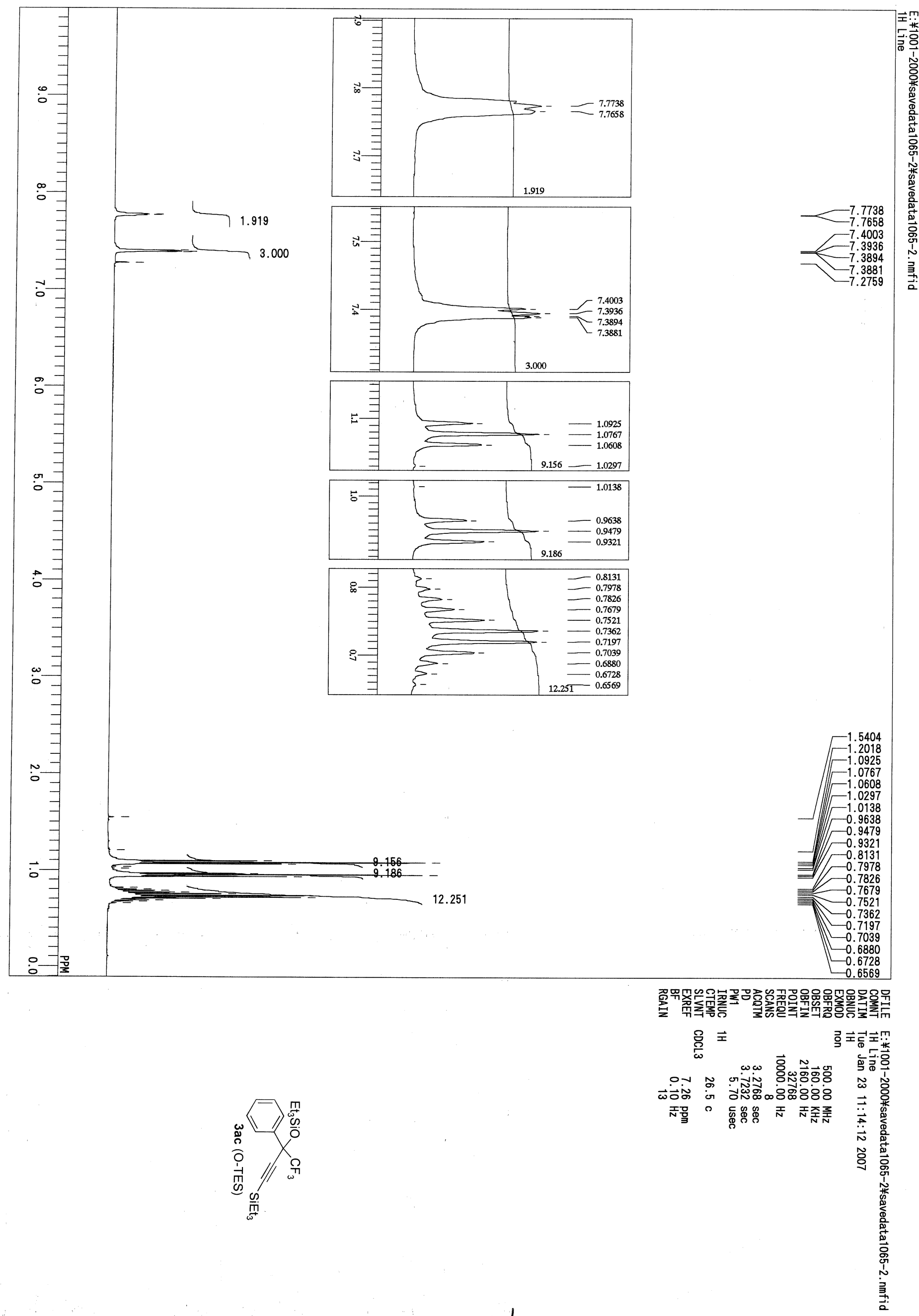


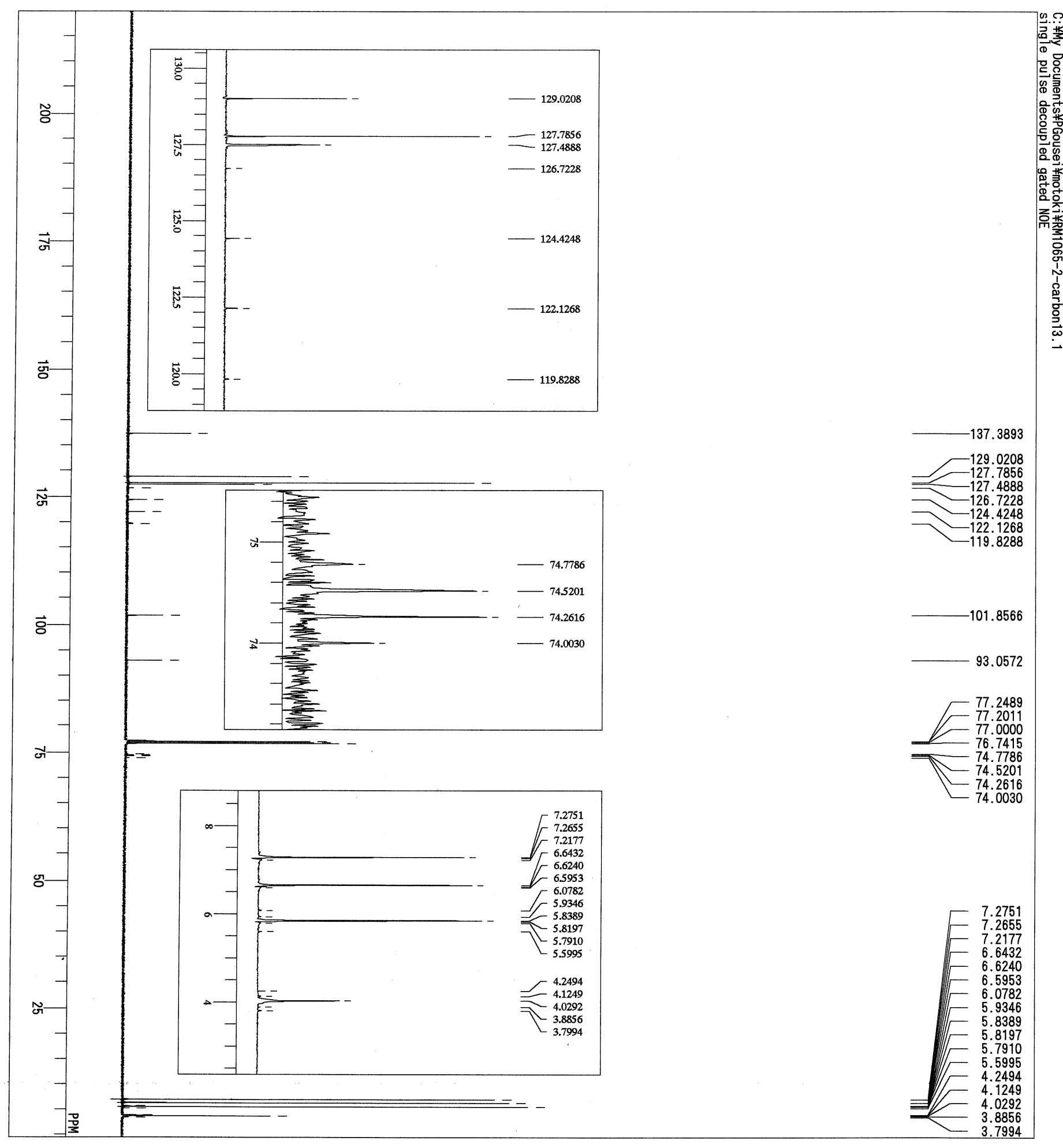

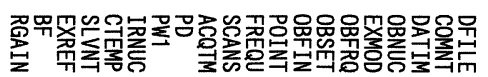

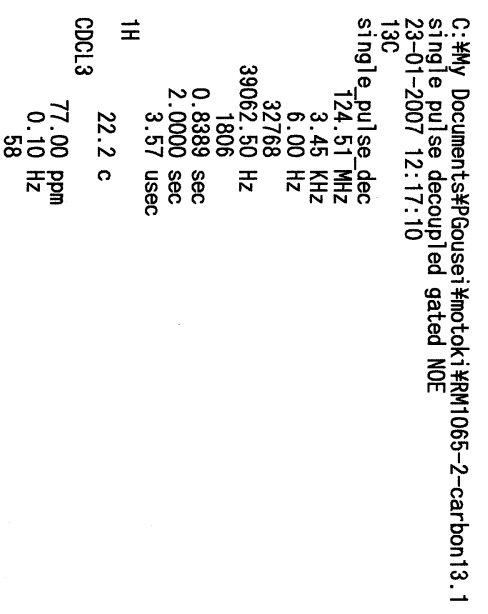




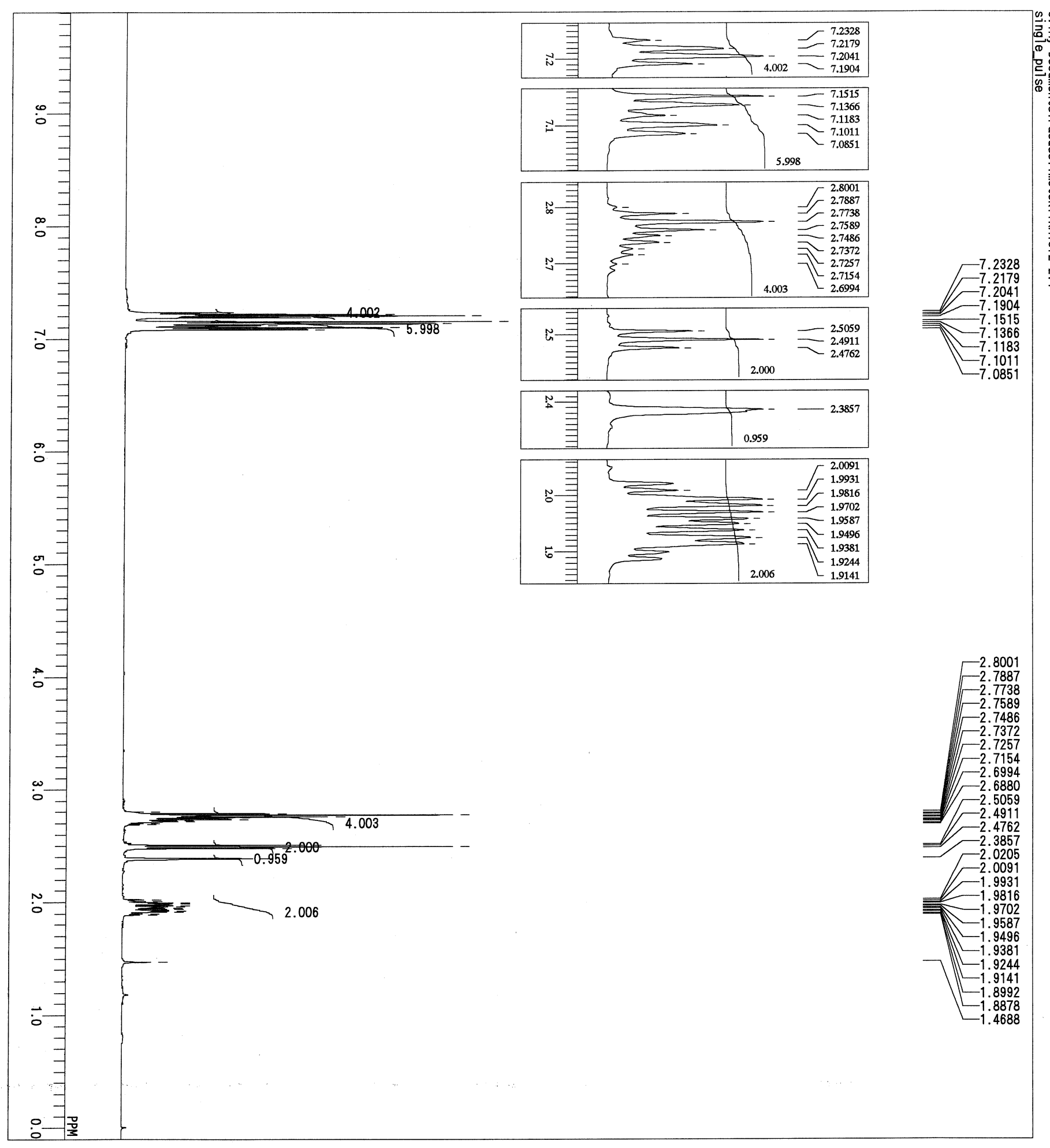

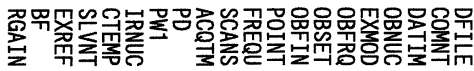
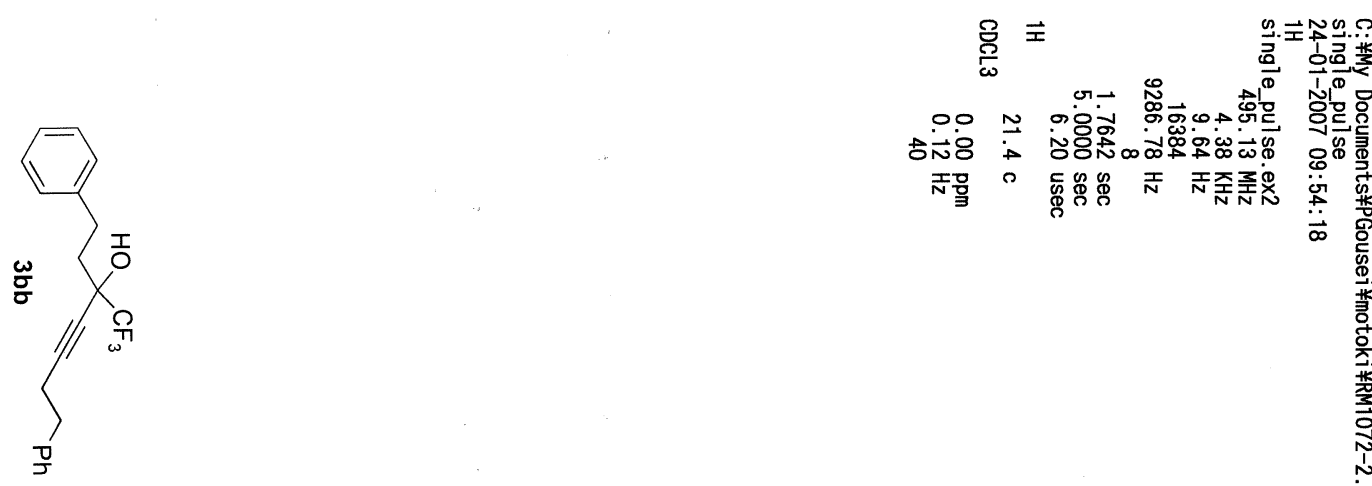


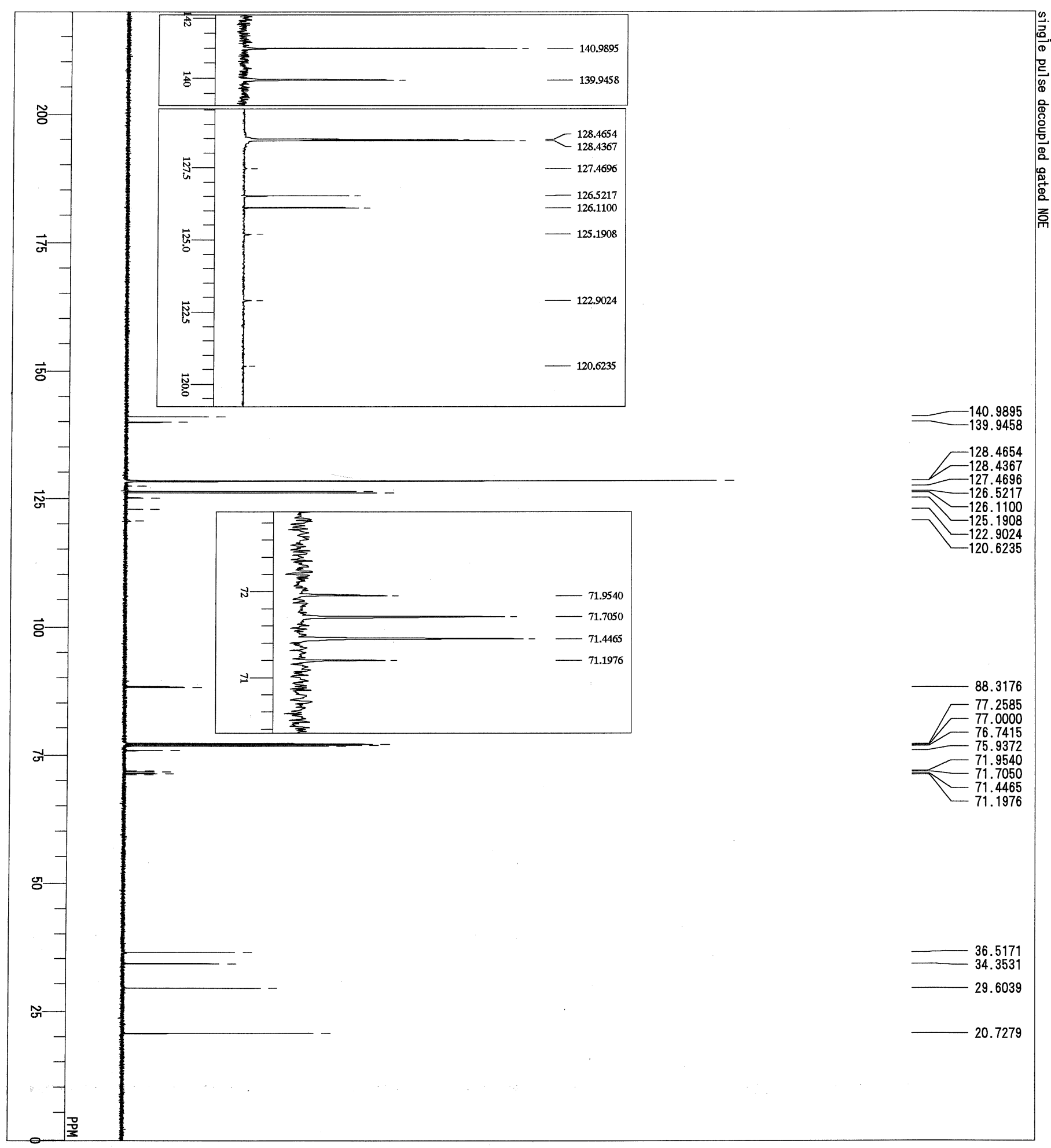

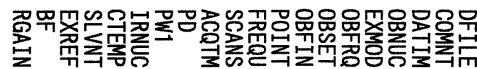

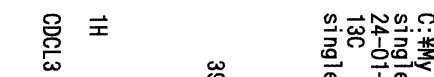
ㄱ No

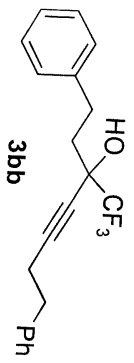

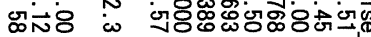

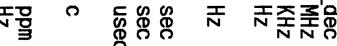

3176 805 


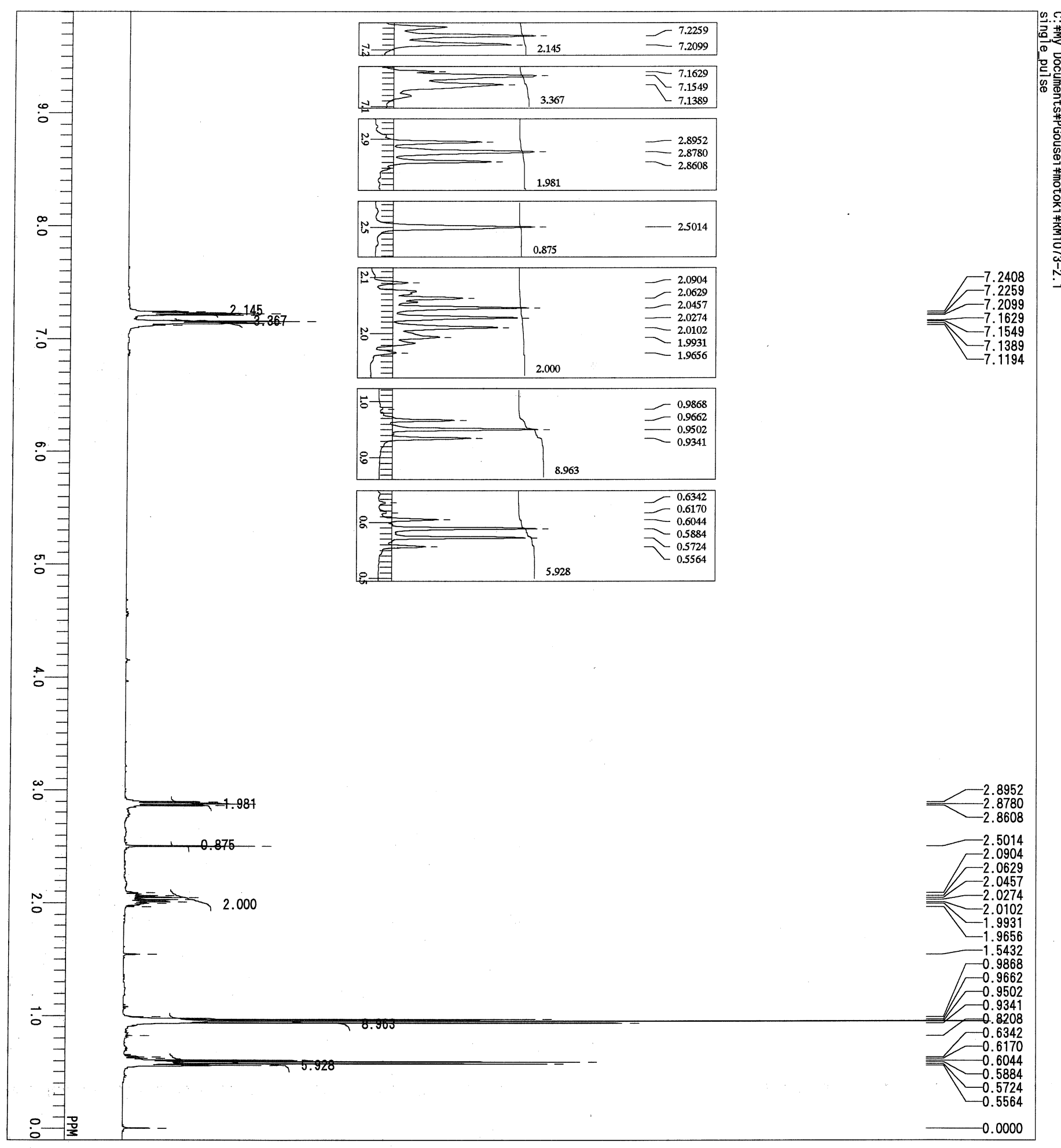

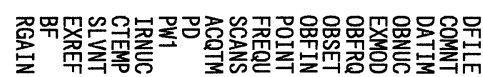
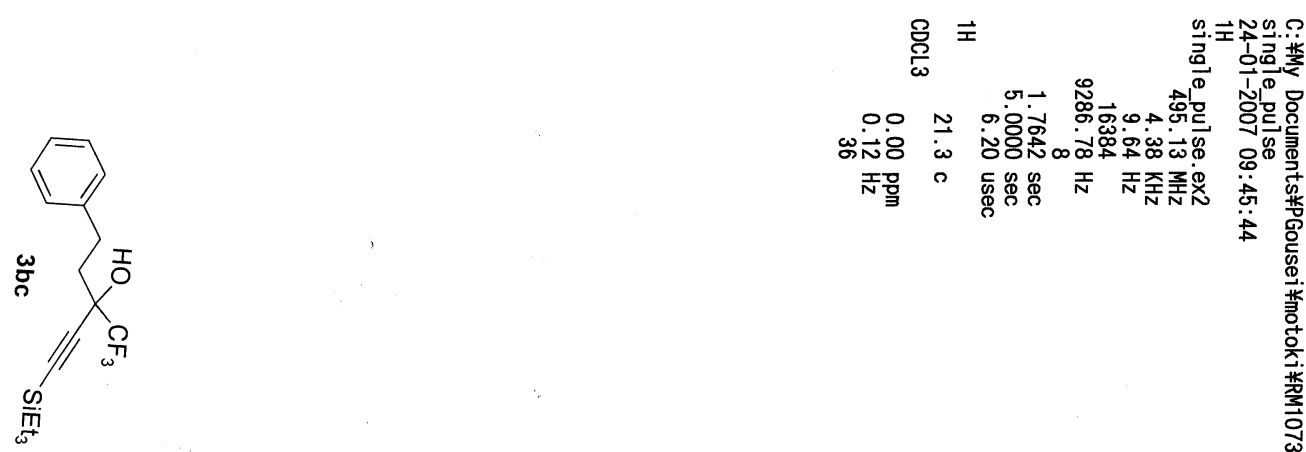

o N

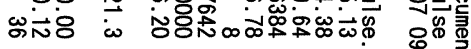

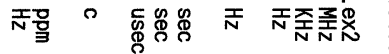













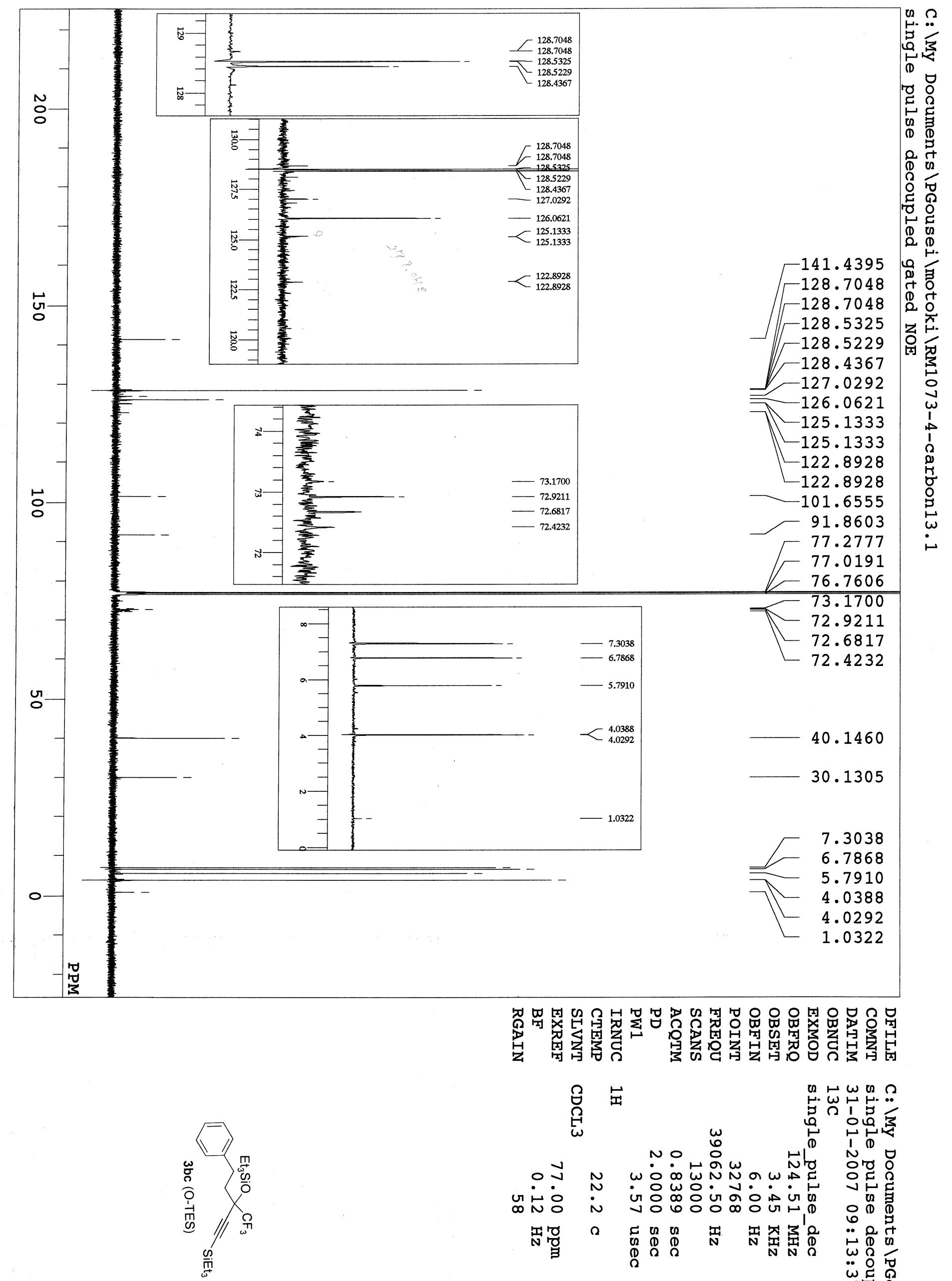

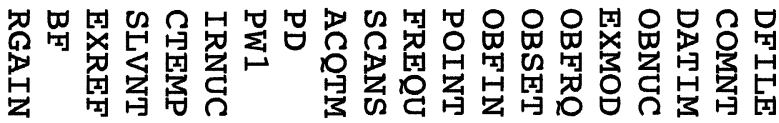

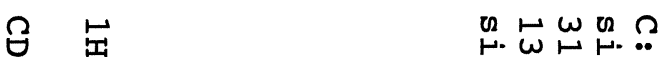
$\stackrel{\overbrace{}}{\leftrightarrow}$

O N $\quad$ NO N N ज诰品

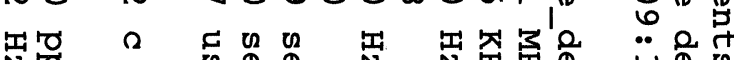

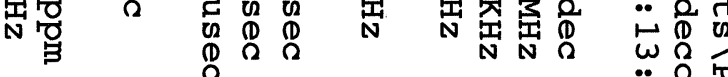
w 0 (1) 

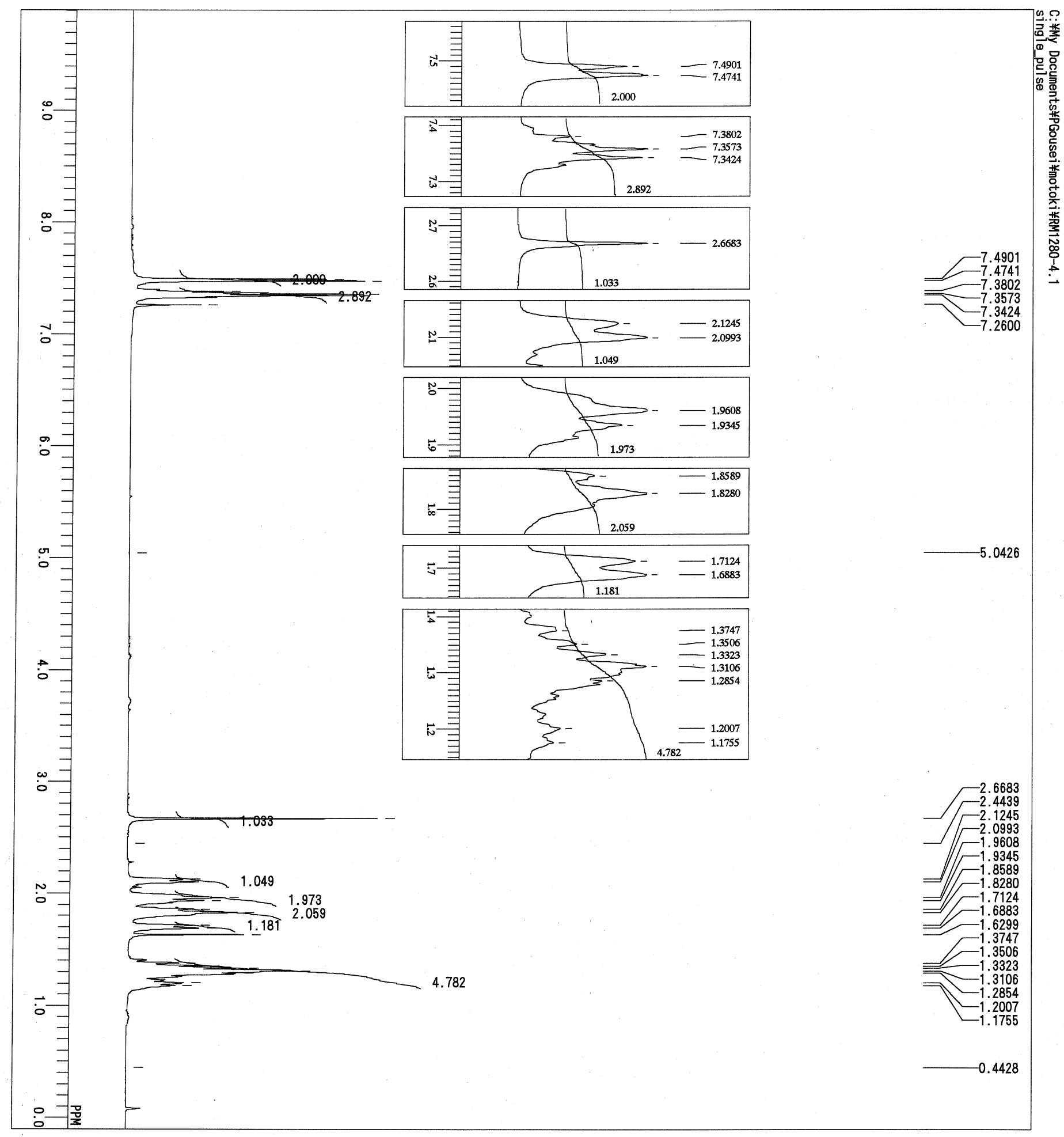

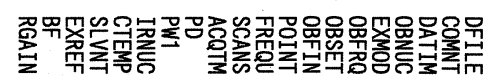
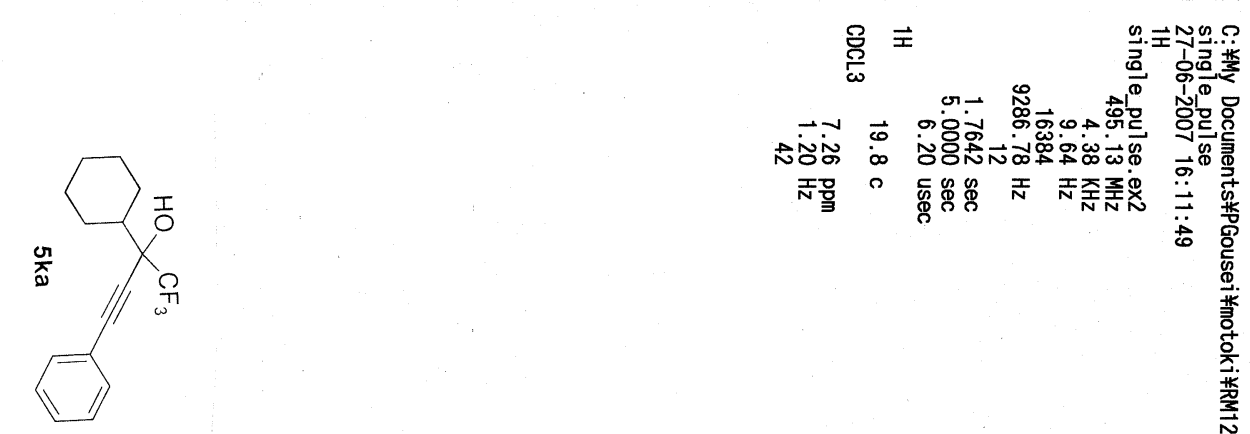

ثีN

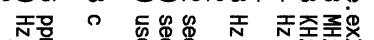

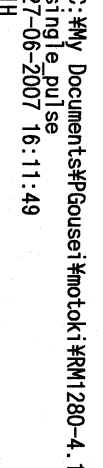




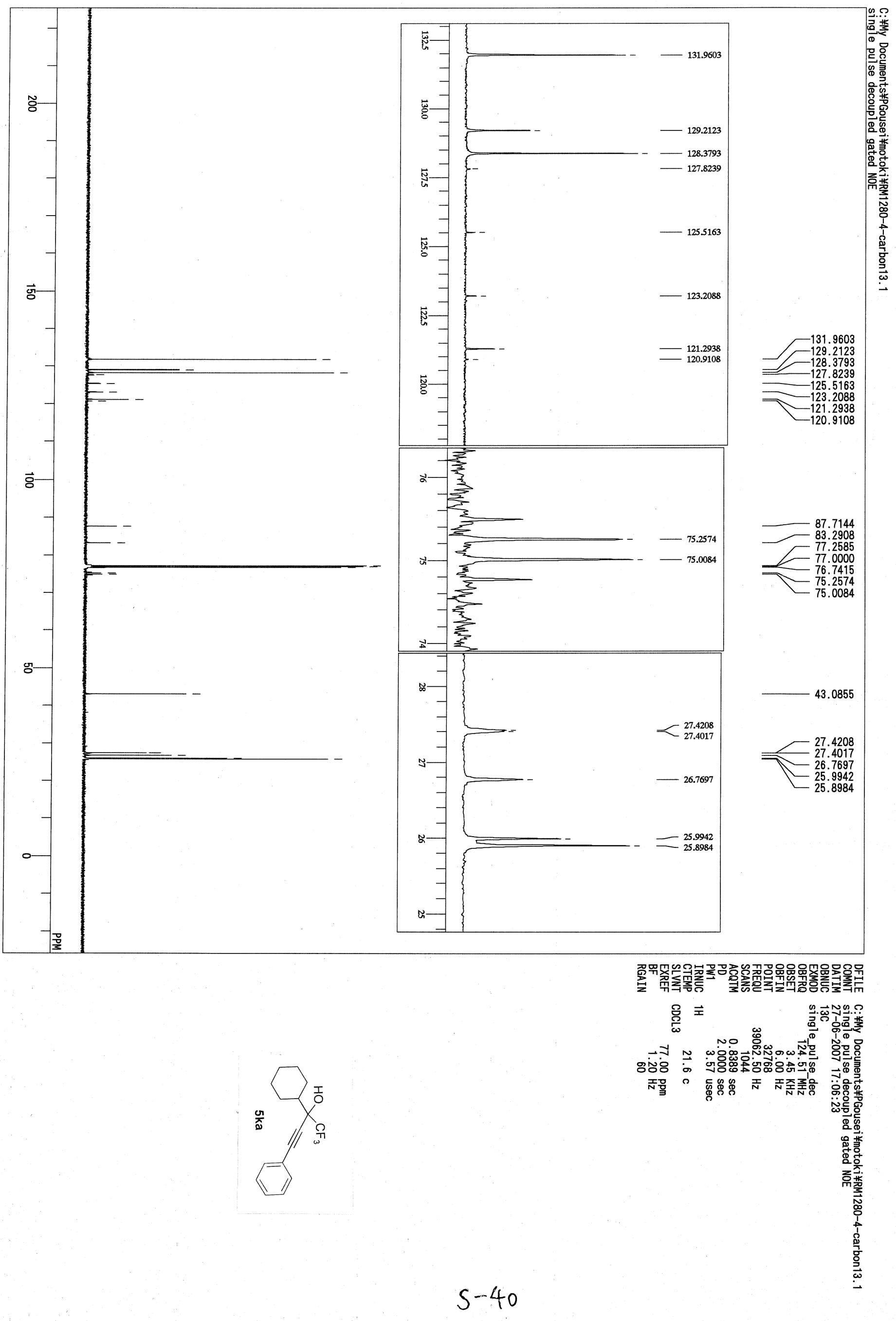

